\title{
Conformal anomaly c-coefficients of superconformal 6d theories
}

\author{
Matteo Beccaria ${ }^{a}$ and Arkady A. Tseytlin ${ }^{b, 1}$ \\ ${ }^{a}$ Dipartimento di Matematica e Fisica Ennio De Giorgi, Università del Salento $\mathscr{G}$ INFN, \\ Via Arnesano, 73100 Lecce, Italy \\ ${ }^{b}$ The Blackett Laboratory, Imperial College, \\ London SW' 2AZ, U.K. \\ E-mail: matteo.beccaria@le.infn.it, tseytlin@imperial.ac.uk
}

ABSTRACT: We propose general relations between the conformal anomaly and the chiral (R-symmetry and gravitational) anomaly coefficients in $6 \mathrm{~d}(1,0)$ superconformal theories. The suggested expressions for the three type B conformal anomaly $c_{i}$-coefficients complement the expression for the type A anomaly a-coefficient found in arXiv:1506.03807. We check them on several examples - the standard $(1,0)$ hyper and tensor multiplets as well as some higher derivative short multiplets containing vector fields that generalize the superconformal $6 \mathrm{~d}$ vector multiplet discussed in arXiv:1506.08727. We also consider a family of higher derivative superconformal $(2,0) 6 \mathrm{~d}$ multiplets associated to $7 \mathrm{~d}$ multiplets in the KK spectrum of $11 \mathrm{~d}$ supergravity compactified on $S^{4}$. In particular, we prove that $(2,0)$ 6d conformal supergravity coupled to 26 tensor multiplets is free of all chiral and conformal anomalies. We discuss some interacting $(1,0)$ superconformal theories, predicting the $c_{i}$-coefficients for the "E-string" theory on multiple M5-branes at $E_{8}$ 9-brane and for the theory describing M5-branes at an orbifold singularity $\mathbb{C}^{2} / \Gamma$. Finally, we elaborate on holographic computation of subleading corrections to conformal anomaly coefficients coming from $R^{2}+R^{3}$ terms in $7 \mathrm{~d}$ effective action, revisiting, in particular, the $(2,0)$ theory case.

KeYwords: AdS-CFT Correspondence, Conformal and W Symmetry, Supergravity Models, Higher Spin Symmetry

ArXiv ePrint: 1510.02685

\footnotetext{
${ }^{1}$ Also at Lebedev Institute, Moscow, Russia.
} 


\section{Contents}

1 Introduction and summary 1

2 Simplest free $(1,0) 6 d$ supermultiplets 5

$\begin{array}{lll}3 & \text { Higher derivative spin } 1 \text { superconformal multiplets } & 7\end{array}$

4 Higher derivative spin 2 superconformal multiplets $\quad 11$

$\begin{array}{ll}4.1 \text { Chiral anomaly coefficients } & 12\end{array}$

$\begin{array}{ll}4.2 \text { Conformal anomaly coefficients } & 14\end{array}$

5 Some interacting $(1,0)$ superconformal theories $\quad 14$

6 6d conformal anomaly from 7d gravitational effective action $\quad 16$

6.1 Quadratic and cubic curvature corrections: linearized approximation $\quad 17$

6.2 Special $(1,0)$ case: Lovelock action 20

$\begin{array}{lll}6.3(2,0) \text { case } & 21\end{array}$

A Conformal anomaly $V^{(1,0)}$ multiplet on Ricci flat background $\quad 23$

B Chiral anomalies of $\mathrm{CSG}_{p}$ supermultiplet $\quad \mathbf{2 4}$

B.1 General $p>2$ case 24

B.2 (2,0) conformal supergravity 26

$\begin{array}{ll}\text { B.3 }(1,0) \text { conformal supergravity } & 27\end{array}$

$\begin{array}{lr}\text { C Casimir energy of 6d supermultiplets } & 28\end{array}$

\section{Introduction and summary}

The aim of this paper is to find general relations between the conformal anomaly and chiral (R-symmetry and gravitational) anomaly coefficients in $6 \mathrm{~d}(1,0)$ superconformal theories. Recently, the expression for the conformal anomaly a-coefficient was suggested in [1]. Here we shall do the same for the conformal anomaly c-coefficients.

In $4 \mathrm{~d} \mathcal{N}=1$ superconformal theories there are linear relations between the a and $\mathrm{c}$ coefficients in the conformal anomaly $[2]^{1}$

$$
A_{4} \equiv(4 \pi)^{2}\langle T\rangle=-\mathrm{a} E_{4}+\mathrm{c} C^{2}
$$

\footnotetext{
${ }^{1}$ Here and below $C$ is the Weyl tensor, $E_{d}$ is the Euler density and we omit total derivative terms in $\langle T\rangle$.
} 
and the coefficients $\alpha, \beta$ in the $\mathrm{U}(1) \mathrm{R}$-symmetry and mixed gravitational anomaly 6 -form polynomial $[3,4]$ (here $R^{2} \equiv R \wedge R$, etc.)

$$
\begin{aligned}
& \mathrm{a}=\frac{3}{32}(3 \alpha-\beta), \quad \mathrm{c}=\frac{1}{32}(9 \alpha-5 \beta), \\
& \mathcal{I}_{6}=\frac{1}{3 !}\left(\alpha c_{1}^{3}+\beta c_{1} \mathrm{p}_{1}\right), \quad c_{1}=\operatorname{tr} F, \quad \mathrm{p}_{1}=-\frac{1}{2} \operatorname{tr} R^{2} .
\end{aligned}
$$

The conformal anomaly of a classically Weyl invariant theory in $6 \mathrm{~d}$ has the following general form $[5-7]$

$$
A_{6} \equiv(4 \pi)^{3}\langle T\rangle=-\mathrm{a} E_{6}+W_{6}, \quad W_{6}=\mathrm{c}_{1} I_{1}+\mathrm{c}_{2} I_{2}+\mathrm{c}_{3} I_{3} .
$$

Here $E_{6}=\epsilon_{6} \epsilon_{6} R R R$ is the Euler density in six dimensions and $W_{6}$ is a combination of the three independent Weyl invariants $I_{1}=C_{a m n b} C^{m p q n} C_{p a b q}, \quad I_{2}=C_{a b m n} C^{m n p q} C_{p q a b}, I_{3}=$ $C_{m n p q} \nabla^{2} C^{m n p q}+\ldots$ (for details see [7]). ${ }^{2}$ Thus, in general, in $6 \mathrm{~d}$ there are 4 independent conformal anomaly coefficients. ${ }^{3}$ Let us note that on a Ricci flat background one has the identities

$$
E_{6}=32\left(2 I_{1}+I_{2}\right), \quad I_{3}=4 I_{1}-I_{2},
$$

so that (1.4) takes the form

$$
\left.A_{6}\right|_{R_{m n}=0}=-\left[\mathrm{a}-\frac{1}{192}\left(\mathrm{c}_{1}+4 \mathrm{c}_{2}\right)\right] E_{6}+\left(\mathrm{c}_{1}-2 \mathrm{c}_{2}+6 \mathrm{c}_{3}\right) I_{1} .
$$

In the presence of $(1,0)$ supersymmetry one expects that the three Weyl invariants $I_{i}$ are bosonic parts of only two possible $6 \mathrm{~d}$ superinvariants, i.e. the coefficients $c_{i}$ should satisfy one linear relation. As suggested by free-theory examples $((1,0)$ scalar and tensor multiplet) [7] and holography based strong-coupling arguments [15], this relation should be ${ }^{4}$

$$
(1,0): \quad q_{1} \equiv \mathrm{c}_{1}-2 \mathrm{c}_{2}+6 \mathrm{c}_{3}=0 .
$$

Then, (1.6) implies that in this case the conformal anomaly on a Ricci flat background is simply proportional to the Euler density (and thus the integrated or scale anomaly vanishes on an asymptotically flat space).

In the case of $(2,0)$ supersymmetry, the invariants $I_{i}$ should be part of a single superinvariant $\mathcal{W}_{6}$ so that $c_{i}$ should be subject to one additional constraint

$$
(2,0): \quad q_{2} \equiv \mathrm{c}_{1}-4 \mathrm{c}_{2}=0, \quad \text { i.e. } \quad \mathrm{c}_{1}=4 \mathrm{c}_{2}=-12 \mathrm{c}_{3} \equiv 96 \mathrm{c} .
$$

\footnotetext{
${ }^{2}$ The sign of $E_{6}$ to be used here (we shall assume Euclidean signature) is opposite to the one in $[7,8]$ but the sign of the a-coefficient will be the same, i.e. negative for standard unitary scalar, spinor or tensor field. We choose not to reverse the sign of a compared to the $4 \mathrm{~d}$ case (1.1), i.e. not to include an extra $(-1)^{d / 2}$ factor (as is done, e.g., in [9] in discussion of free energy on a sphere) so that the a-coefficient is always directly proportional to the coefficient of the logarithmic UV divergence (see, e.g., [10]).

${ }^{3}$ The Weyl anomaly may be related to the correlation functions of the stress tensor on a flat background [11]. In $4 \mathrm{~d}$ the a-coefficient is related to the 3 -point function $\langle T T T\rangle$ and $\mathrm{c}$ is proportional to the coefficient in the 2-point function $\langle T T\rangle$. In $6 \mathrm{~d}$, the a-coefficient is related to the 4-point function $\langle T T T T\rangle$. The coefficient $c_{3}$ is related to $\langle T T\rangle$, while $c_{1}$ and $c_{2}$ are related to the two free parameters in $\langle T T T\rangle$ (in $6 \mathrm{~d}$ this 3-point function has three parameters, but one of them is related to the 2-point function by a conformal Ward identity [12-14]).

${ }^{4}$ This relation appeared also in other contexts in [16-18].
} 
In this case there is only one independent c-coefficient, i.e.

$$
(2,0): \quad A_{6}=-\mathrm{a} E_{6}+\mathrm{c} \mathcal{W}_{6}, \quad \mathcal{W}_{6} \equiv 96 I_{1}+24 I_{2}-8 I_{3} .
$$

This expectation is supported by the results for the anomaly of a free $(2,0)$ tensor multiplet in [7] and for the strong coupling (large $N$ ) limit of interacting $(2,0)$ theory [19]. Then on Ricci flat background one gets from (1.6)

$$
(2,0):\left.\quad A_{6}\right|_{R_{m n}=0}=(\mathrm{c}-\mathrm{a}) E_{6},
$$

which is the analog of the familiar $\left.A_{4}\right|_{R_{m n}=0}=(\mathrm{c}-\mathrm{a}) E_{4}$ relation in $4 \mathrm{~d}$ following from (1.1).

Since like $4 \mathrm{~d}$ anomalies the $6 \mathrm{~d}$ anomalies form a supersymmetry multiplet [20-22] one expects to find linear relations between their coefficients analogous to (1.2). The $6 \mathrm{~d}$ chiral (SU(2) R-symmetry and gravitational) anomaly 8-form polynomial has the following general structure [23-25]

$$
\begin{aligned}
& \mathcal{I}_{8}=\frac{1}{4 !}\left(\alpha c_{2}^{2}+\beta c_{2} \mathrm{p}_{1}+\gamma \mathrm{p}_{1}^{2}+\delta \mathrm{p}_{2}\right), \\
& c_{2}=\operatorname{tr} F^{2}, \quad \mathrm{p}_{2}=-\frac{1}{4} \operatorname{tr} R^{4}+\frac{1}{8}\left(\operatorname{tr} R^{2}\right)^{2},
\end{aligned}
$$

where $c_{1}$ and $\mathrm{p}_{1}$ are defined as in (1.3) and $\vec{\alpha}=(\alpha, \beta, \gamma, \delta)$ are numerical coefficients. ${ }^{5}$

In [1] an expression for the a-coefficient in $(1.4)$ in $(1,0)$ superconformal theory written in terms of the chiral anomaly coefficients $\vec{\alpha}$ was found by determining the 4 parameters $\vec{k}$ in the expected linear relation $\mathrm{a}=\vec{k} \cdot \vec{\alpha}$ from several explicit examples: ${ }^{6}$

$$
\mathrm{a}=-\frac{1}{72}\left(\alpha-\beta+\gamma+\frac{3}{8} \delta\right)
$$

Equivalently, this may be written as

$$
\mathrm{a}=\frac{16}{7}\left(\alpha-\beta+\gamma+\frac{3}{8} \delta\right) \mathrm{a}_{\mathrm{T}}, \quad \mathrm{a}_{\mathrm{T}} \equiv \mathrm{a}\left(T^{(2,0)}\right)=-\frac{7}{1152},
$$

where $\mathrm{a}_{\mathrm{T}}$ is the value of a for the $(2,0)$ tensor multiplet [7] for which $\vec{\alpha}=\left(1, \frac{1}{2}, \frac{1}{8},-\frac{1}{2}\right)$.

Here we shall follow the same strategy to find the analogs of (1.13) for the two independent conformal anomaly $c_{i}$-coefficients, e.g., $c_{1}$ and $c_{2}$ (with $c_{3}$ then given by (1.7)). Using as input some known examples we suggest the following expressions ${ }^{7}$

$$
\begin{aligned}
& \mathrm{c}_{1}=-\frac{4}{3}(\alpha-\beta)-\frac{52}{27} \gamma-\frac{31}{27} \delta, \quad \mathrm{c}_{2}=-\frac{1}{3}(\alpha-\beta)-\frac{5}{27} \gamma-\frac{23}{108} \delta, \\
& \mathrm{c}_{3}=-\frac{1}{6}\left(\mathrm{c}_{1}-2 \mathrm{c}_{2}\right) .
\end{aligned}
$$

\footnotetext{
${ }^{5}$ The R-symmetry gauge bundle forms $c_{1}$ and $c_{2}$ should not be confused with the conformal anomaly coefficients $\mathrm{c}_{1}, \mathrm{c}_{2}$.

${ }^{6}$ This relation (and similar relations below) between chiral and conformal anomalies applies for $(1,0)$ supermultiplets; for $(0,1)$ ones one should flip the overall sign as conformal anomaly is not sensitive to chirality (of fermions and antisymmetric tensors) while chiral anomalies are.

${ }^{7}$ It remains to see if these relations may be used to interpret (at fixed points) the RG flow $C$-functions discussed in [26] in terms of the conformal anomaly coefficients.
} 
Like a in (1.13) all $\mathrm{c}_{i}$ then depend on $\alpha, \beta$ only through $\alpha-\beta$, suggesting that $c_{2}^{2}-c_{2} \mathrm{p}_{1}$ combination in the anomaly $(1.11)$ is part of $(1,0)$ superinvariant related to conformal anomaly. Also, we observe that

$$
c_{1}-4 c_{2}=-\frac{8}{27}(4 \gamma+\delta)
$$

so that in the special $(2,0)$ supersymmetry case when $(1.8)$ is expected to be satisfied we should have

$$
(2,0): \quad \gamma=-\frac{1}{4} \delta .
$$

This is consistent with the fact that $\mathrm{p}_{2}-\frac{1}{4} \mathrm{p}_{1}^{2}$ in (1.11) should form part of associated superinvariant. ${ }^{8}$ Also, in the $(2,0)$ case the coefficients $\beta$ and $\gamma$ in $(1.11)$ appear to be related by

$$
(2,0): \quad \beta=4 \gamma .
$$

Then it follows that in the $(2,0)$ case (see (1.8), (1.13), (1.15), (1.17))

$$
\begin{aligned}
& (2,0): \quad \mathrm{a}=-\frac{1}{72}\left(\alpha+\frac{9}{8} \delta\right), \quad \mathrm{c}=-\frac{1}{72}\left(\alpha+\frac{3}{2} \delta\right), \\
& \mathrm{c}_{1}=4 \mathrm{c}_{2}=-12 \mathrm{c}_{3}=96 \mathrm{c} \text {, } \\
& \mathrm{a}-\mathrm{c}=\frac{1}{192} \delta \text {. }
\end{aligned}
$$

The relation (1.21) allows one to determine c from the knowledge of a-coefficient and the gravitational anomaly coefficient $\delta$.

Below, we shall be discussing 1-loop conformal anomalies in higher derivative theories. ${ }^{9}$ In general, the conformal anomaly in the trace of stress tensor on a curved background is related to a logarithmic UV divergence and thus it is determined by the corresponding heat kernel (Seeley) coefficient. This relation is "kinematical" and is expected to apply regardless the order of the differential operator involved or metric signature (and thus also other issues like unitarity). One important assumption will be the existence of Weylcovariant generalisations of flat-space conformal higher derivative operators that have the correct gauge invariance at least on conformally flat and Ricci-flat backgrounds (cf. [27]). ${ }^{10}$ As for chiral anomalies, they are essentially the same (modulo ghost counting, etc.) for the standard and higher-derivative operators (see, e.g., [24, 28-30]).

The structure of the rest of this paper is as follows. In section 2 we shall summarize the results for conformal and chiral anomalies of the standard $(1,0)$ hyper $S^{(1,0)}$ and tensor $T^{(1,0)}$ multiplets and also for the $6 \mathrm{~d}$ conformal analog $V^{(1,0)}$ of the Maxwell multiplet which contains 4-derivative vector, 3 -derivative spinor and 2-derivative scalar fields. This will provide evidence for the relation (1.15).

\footnotetext{
${ }^{8}$ This 8-form multiplied by $C_{3}$-potential is bosonic part of a $11 \mathrm{~d} \epsilon_{11} C_{3} R R R R$ superinvariant.

${ }^{9}$ In general, the properties of anomalies in higher-derivative theories (and comparison with their derivation in the standard unitary theories) deserves a thorough investigation that we postpone till future work.

${ }^{10} \mathrm{We}$ will considering only universal, i.e. regularisation (or scheme) independent terms in $6 \mathrm{~d}$ conformal anomaly that cannot be changed by adding local counterterms.
} 
In section 3 we shall consider a family $V_{p}^{(1,0)}$ of higher derivative "massive" $(1,0)$ conformal multiplets generalizing the "massless" (gauge-invariant) vector multiplet $V^{(1,0)}$. They appear in tensor product of $p$ copies of $S^{(1,0)}$ scalar multiplets. We shall independently compute their chiral and conformal anomalies and provide an additional check of (1.15).

In section 4 we shall compute the anomalies of a family $\mathrm{CSG}_{p}$ of $(2,0) 6 \mathrm{~d}$ multiplets that generalize the $(2,0)$ conformal supergravity multiplet (corresponding to $p=2$ case). These are associated to $7 \mathrm{~d}$ multiplets that appear in the KK spectrum of $11 \mathrm{~d}$ supergravity compactified on $S^{4}$, i.e. appear in tensor product of $p$ copies of $T^{(2,0)}$ tensor multiplets. We verify the relation (1.14) and apply (1.19) to compute the corresponding c-anomaly. We show, in particular, that the system of $(2,0)$ conformal supergravity coupled to 26 tensor multiplets is chiral and conformal anomaly free.

In section 5 we shall apply the relations (1.15) to compute $c_{i}$-coefficients of some interacting $(1,0)$ superconformal $6 \mathrm{~d}$ theories for which the chiral anomaly coefficients are known: the E-string theory on multiple M5-branes at $E_{8}$ 9-brane and the theory on M5-branes at an orbifold singularity $\mathbb{C}^{2} / \Gamma$. We observe some relations between the $c_{i}$-coefficients that indirectly support the consistency of our suggested expressions (1.15).

Finally, in section 6 we shall consider the AdS/CFT based computation of subleading corrections to the $6 \mathrm{~d}$ conformal anomaly coefficients coming from $R^{2}+R^{3}$ terms in $7 \mathrm{~d}$ effective action discussing, in particular, the supersymmetry constraints (1.7) and (1.8) and $1 / N^{2}$ corrections to conformal anomaly in $(2,0)$ theory.

In appendix A we shall compute the conformal anomaly coefficients for the higherderivative vector multiplet $V^{(1,0)}$ on a Ricci flat background. Appendix B will be devoted to the computation of chiral anomaly coefficients for the $\mathrm{CSG}_{p}$ multiplets. We shall also give a separate discussion of the anomalies of the $(2,0)$ and $(1,0)$ conformal supergravities. In appendix C we will propose an expression for the $S^{5}$ Casimir energy $E_{c}$ for $(1,0)$ multiplets given like (1.13), (1.15) by a linear combination of the chiral anomaly coefficients.

\section{Simplest free $(1,0) 6 d$ supermultiplets}

The conformal anomalies of free 2-derivative scalar $\varphi$, Majorana-Weyl (MW) spinor $\psi$ and (anti)selfdual rank 2 tensor $T$ were computed in [7]. Combining these fields into $(1,0)$ scalar and tensor and $(2,0)$ tensor supermultiplets (we indicate physical fields only and their chirality)

$$
\begin{aligned}
& S^{(1,0)}=4 \varphi+2 \psi^{-}, \quad T^{(1,0)}=\varphi+2 \psi^{-}+T^{-}, \\
& T^{(2,0)}=S^{(1,0)}+T^{(1,0)}=5 \varphi+4 \psi^{-}+T^{-},
\end{aligned}
$$

we get the corresponding values of a and $c_{i}$ in (1.4). The results are summarized in table 1 . We observe that for $S^{(1,0)}$ and $T^{(1,0)}$ the relation (1.7) is satisfied, while for $T^{(2,0)}$ we have also the relation (1.8). It is reasonable to assume that (1.7) follows from the requirement of $(1,0)$ supersymmetry on the Weyl super-cocycle and should thus be true in all $(1,0)$ cases, free or interacting. This is also consistent also with what is implied by holographic computation of conformal anomaly $[15,31]$. 


\begin{tabular}{|c|cccc|}
\hline & $\mathrm{a}$ & $\mathrm{c}_{1}$ & $\mathrm{c}_{2}$ & $\mathrm{c}_{3}$ \\
\hline$\varphi$ & $-\frac{1}{72576}$ & $-\frac{1}{540}$ & $\frac{1}{3024}$ & $\frac{1}{2520}$ \\
$\psi^{-}$ & $-\frac{191}{1451520}$ & $-\frac{2}{135}$ & $-\frac{1}{630}$ & $\frac{1}{504}$ \\
$T^{-}$ & $-\frac{221}{40320}$ & $-\frac{143}{540}$ & $-\frac{1189}{15120}$ & $\frac{1}{56}$ \\
\hline$S^{(1,0)}$ & $-\frac{11}{34560}$ & $-\frac{1}{27}$ & $-\frac{1}{540}$ & $\frac{1}{180}$ \\
$T^{(1,0)}$ & $-\frac{199}{34560}$ & $-\frac{8}{27}$ & $-\frac{11}{135}$ & $\frac{1}{45}$ \\
\hline$T^{(2,0)}$ & $-\frac{7}{1152}$ & $-\frac{1}{3}$ & $-\frac{1}{12}$ & $\frac{1}{36}$ \\
\hline
\end{tabular}

Table 1. Conformal anomaly coefficients for free fields and supermultiplets.

Let us also consider the standard $6 \mathrm{~d}(1,0)$ supersymmetric vector multiplet $V_{\mathrm{s}}^{(1,0)}$ (which includes the 2-derivative Maxwell field $V$ and thus is not superconformal) and also the higher-derivative superconformal $6 \mathrm{~d}(1,0)$ vector multiplet $V^{(1,0)}$ that includes the 4-derivative vector $V^{(4)}$ and 3-derivative spinor $\psi^{(3)}[8,32]$

$$
V_{\mathrm{s}}^{(1,0)}=2 \psi^{+}+V, \quad V^{(1,0)}=3 \varphi+2 \psi^{(3),+}+V^{(4)} .
$$

The supersymmetric vector multiplet $V_{\mathrm{s}}^{(1,0)}$ may be appearing in low-energy limit of a spontaneously broken $6 \mathrm{~d}$ superconformal theory (e.g., from $\phi F_{\mu \nu}^{2}+\ldots$ with $\langle\phi\rangle=$ const). While the conformal anomaly coefficients are not defined for non-conformal $V_{\mathrm{s}}^{(1,0)}$ multiplet, for $V^{(1,0)}$ one finds

$$
V^{(1,0)}: \quad \mathrm{a}=\frac{251}{34560}, \quad \mathrm{c}_{1}=\frac{19}{27}, \quad \mathrm{c}_{2}=\frac{91}{540}, \quad \mathrm{c}_{3}=-\frac{11}{180} .
$$

Here a can be determined [8] from the value of the conformal anomaly on $S^{6}$ - either directly in $6 \mathrm{~d}$ or using the $\mathrm{AdS}_{7}$ based method [33-35]. The values of $c_{i}$ can be computed starting with the curved space kinetic operators given in [8] and are again consistent with the constraint (1.7) (see appendix A).

Let us now consider the corresponding chiral anomaly coefficients in (1.11). The only fields that contribute to the chiral anomalies are the Weyl fermions and self-dual tensors (their values can be found in [24]). Also, the chiral anomaly contribution of 3-derivative fermion $\psi^{(3)}$ is the same as of the standard 1-derivative fermion $\psi$. This leads to the coefficients summarized in table 2 where we also give the results for the a coefficient verifying the relation (1.14) of [1]. Few explanations are in order. The values for $T^{(2,0)}$ in $(2.1)$ are the sums of the values for $S^{(1,0)}$ and $T^{(1,0)}$. The chiral anomalies of $V_{\mathrm{s}}^{(1,0)}$ and $V^{(1,0)}$ are the same as they come only from the fermions and are not sensitive to extra $\partial^{2}$ in the kinetic term of $\psi^{(3)}$ in (2.2). The values of $\alpha, \beta$ for $S^{(1,0)}$ are zero since the corresponding fermions in (2.1) are singlets of $\mathrm{SU}(2)_{R}$. The fermions of $T^{(1,0)}$ form a doublet of $\mathrm{SU}(2)_{R}$ as are the fermions of $V_{\mathrm{s}}^{(1,0)}$ and $V^{(1,0)}$ but their $\alpha, \beta$ coefficients differ in sign due to different chirality assignments in (2.1), (2.2) (the antisymmetric tensor of $T^{(1,0)}$ does not contribute 


\begin{tabular}{|c|c|c|c|c|c|}
\hline & $\alpha$ & $\beta$ & $\gamma$ & $\delta$ & $\mathrm{a} / \mathrm{a}_{\mathrm{T}}$ \\
\hline$S^{(1,0)}$ & 0 & 0 & $\frac{7}{240}$ & $-\frac{1}{60}$ & $\frac{11}{210}$ \\
\hline$T^{(1,0)}$ & 1 & $\frac{1}{2}$ & $\frac{23}{240}$ & $-\frac{29}{60}$ & $\frac{199}{210}$ \\
\hline$T^{(2,0)}$ & 1 & $\frac{1}{2}$ & $\frac{1}{8}$ & $-\frac{1}{2}$ & 1 \\
\hline$V_{\mathrm{s}}^{(1,0)}$ & -1 & $-\frac{1}{2}$ & $-\frac{7}{240}$ & $\frac{1}{60}$ & - \\
\hline$V^{(1,0)}$ & -1 & $-\frac{1}{2}$ & $-\frac{7}{240}$ & $\frac{1}{60}$ & $-\frac{251}{210}$ \\
\hline
\end{tabular}

Table 2. Chiral anomaly coefficients and a coefficient (in units of $T^{(2,0)}$ value).

to $\alpha, \beta$ as it is singlet of R-symmetry). The gravitational anomalies $\gamma, \delta$ of $S^{(1,0)}$ and of $V_{\mathrm{S}}^{(1,0)}$ or $V^{(1,0)}$ are the same up to sign as they come from fermions of opposite chirality (for $T^{(1,0)}$ there are also additional contributions of antisymmetric tensor).

It was noted in [1] that the relation (1.14) formally applied to the standard (scale invariant but not conformally invariant vector multiplet $V_{\mathrm{s}}^{(1,0)}$ for which a is not defined) leads to the value $\mathrm{a}=-\frac{251}{210} \mathrm{a}$ which has the opposite sign to a of $S^{(1,0)}$ and $T^{(1,0)}$. As was observed in [8], this value corresponds, in fact, to the higher-derivative superconformal multiplet $V^{(1,0)}$. It now appears that the reason for this curious observation is simply of technical nature - it follows from the fact that the chiral anomalies of $V_{\mathrm{s}}^{(1,0)}$ and $V^{(1,0)}$ are the same, and $V^{(1,0)}$ being higher-derivative (non-unitary) happens to have an opposite sign of a-coefficient compared to the one of the standard unitary scalar and tensor multiplets.

Turning now to the conformal anomaly $c_{i}$-coefficients we observe that their values in table 1 and (2.3) are indeed consistent with the relations (1.15), (1.7) where $\alpha, \beta, \gamma, \delta$ are given by table 2 . The values for the three multiplets $S^{(1,0)}, T^{(1,0)}$ and $V^{(1,0)}$ are sufficient to fix 3 out of 4 a priori unknown coefficients in the relations (1.15) between $c_{i}$ and chiral anomaly coefficients.

\section{Higher derivative spin 1 superconformal multiplets}

To provide further examples of anomaly computation for $(1,0)$ superconformal multiplets and to support the relations (1.15) let us now consider a family higher derivative analogs of the vector multiplet $V^{(1,0)}$. These multiplets, that we will denote as $\mathrm{V}_{p}^{(1,0)}, p \geq 2$, will contain scalars, spinors and vectors with higher-derivative kinetic terms. The $p=2$ case will correspond to $V^{(1,0)}$ discussed above. From the point of view of $\operatorname{OSp}(2,6 \mid 2)$ representations [36-38] the hypermultiplet $S^{(1,0)}$ is a doubleton ultra-short representation [39]. ${ }^{11}$ Additional (possibly massive) conformal representations are obtained from the tensor product of $p$ copies of $S^{(1,0)}$. The resulting multiplets $\mathrm{V}_{p}^{(1,0)}$ are still short but have maximal spin equal to 1 instead of $\frac{1}{2}$ as in the $S^{(1,0)}$ case. The structure of these multiplets was worked out in [41] and is summarized in table 3.

\footnotetext{
${ }^{11}$ In general, the relevant superconformal algebras are $\operatorname{OSp}\left(8^{*} \mid \mathcal{N}\right)$ with compact R-symmetry $\operatorname{USp}(\mathcal{N})$. Our notation $\operatorname{OSp}(2,6 \mid 2)$ implicitly exploits the isomorphism $S O^{*}(8)=\mathrm{SO}(2,6)[40]$.
} 


\begin{tabular}{|cccc|}
\hline & $\mathrm{SO}(6)$ & $\mathrm{SU}(2)_{R}$ & $\Delta$ \\
\hline$\varphi$ & $(0,0,0)$ & $\mathbf{p}+\mathbf{1}$ & $2 p$ \\
$\psi^{+}$ & $\left(\frac{1}{2}, \frac{1}{2}, \frac{1}{2}\right)$ & $\mathbf{p}$ & $2 p+\frac{1}{2}$ \\
$V_{m}$ & $(1,0,0)$ & $\mathbf{p}-\mathbf{1}$ & $2 p+1$ \\
$\psi^{-}$ & $\left(\frac{1}{2}, \frac{1}{2},-\frac{1}{2}\right)$ & $\mathbf{p}-\mathbf{2}$ & $2 p+\frac{3}{2}$ \\
$\varphi^{\prime}$ & $(0,0,0)$ & $\mathbf{p}-\mathbf{3}$ & $2 p+2$ \\
\hline
\end{tabular}

Table 3. Short multiplets $\mathrm{V}_{p}^{(1,0)}$ of $\operatorname{OSp}(2,6 \mid 2)$ that appear in the product of $p$ copies of $(1,0)$ doubleton (hypermultiplet).

Here $\Delta$ is the scaling dimension of the conformal group $\mathrm{SO}(2,6)$ related to the canonical dimension of the corresponding $6 \mathrm{~d}$ field $\Phi$ by $\operatorname{dim} \Phi=6-\Delta$. We indicated also the $\operatorname{SU}(2)$ R-symmetry representations. ${ }^{12} \psi^{ \pm}$are positive/negative chirality MW spinors while $\varphi$ and $\varphi^{\prime}$ are scalars. The vector $V_{m}$ is non-gauge ("massive") one for $p>2$ but has the standard gauge invariance for $p=2$.

The $p=2$ case of $\mathrm{V}_{p}^{(1,0)}$ is thus $V^{(1,0)}$ in $(2.2)$. Note also that the formal $p=1$ case of field content in table 3 is the same as the scalar multiplet $S^{(1,0)}$ in $(2.1)$ but with the opposite chirality of the fermion, i.e. $\mathrm{V}_{1}^{(1,0)}=S^{(0,1)}$. Thus the chiral anomalies of $\mathrm{V}_{1}^{(1,0)}$ will be opposite in sign to the anomalies of $S^{(1,0)}$. In what follows we shall assume that $p>1$, as required by the actual construction of $\mathrm{V}_{p}^{(1,0)}$ as a tensor product.

From the canonical dimensions of the fields one can determine the power of derivatives in kinetic terms in the corresponding 6d Lagrangian

$$
\mathcal{L}=\varphi \square^{2 p-3} \varphi+\bar{\psi}^{+} \not \partial^{4 p-5} \psi^{+}+V_{m} \square^{2 p-2} V_{m}+\bar{\psi}^{-} \not^{4 p-3} \psi^{-}+\varphi^{\prime} \square^{2 p-1} \varphi^{\prime}
$$

where each field is assumed to transform under $\mathrm{SU}(2)_{R}$ according to representations in table $3 .^{13}$

Let us first compute the corresponding chiral $\mathrm{SU}(2)_{R}$ and gravitational anomalies. The gravitational anomaly coefficients $\gamma, \delta$ in (1.11) here get contributions from the chiral fermions $\psi^{+}$and $\psi^{-}$and do not depend on powers of derivatives in the kinetic terms, i.e. are the same as for the usual $\not \partial$ fermions. As we have $p$ of $\psi^{+}$fermions and $p-2$ of $\psi^{-}$ fermions the total contribution is as of $p-(p-2)=2$ positive-chirality fermions, i.e. it is the same as for the standard vector multiplet or for $V^{(1,0)}$ in (2.2) (see table 2). Thus the

\footnotetext{
${ }^{12} \mathbf{p}$ stands for a representation of dimension $p$; fields with negative $\mathrm{SU}(2)_{R}$ dimensions should be dropped. For a field in $p$-dimensional representation of $\mathrm{SU}(2)_{R}$ the $d$ values of the $R$-charge are $-\frac{p-1}{2}+k, \quad k=$ $0, \ldots, p-1$.

${ }^{13}$ From the kinetic terms we can compute the corresponding numbers of dynamical d.o.f. for bosons and fermions $(p>2) \quad \nu_{b}=(p+1)(2 p-3)+(p-3)(2 p-1)+6(p-1)(2 p-2)=4(2 p-3)(2 p-1)$, $\nu_{f}=-2[p(4 p-5)+(p-2)(4 p-3)]=-4(2 p-3)(2 p-1)$, so that $\nu_{b}+\nu_{f}=0$. The above expressions are formally true also for $p=2$ when the vector is a gauge-invariant one but there is no second scalar $\varphi^{\prime}$ and this has the same effect as subtracting the gauge mode of $V_{m}$.
} 
$\gamma, \delta$ coefficients are $p$-independent

$$
\mathrm{V}_{p}^{(1,0)}: \quad \gamma=-\frac{7}{240}, \quad \delta=\frac{1}{60} .
$$

The coefficients $\alpha$ and $\beta$ in (1.11) are proportional to the sums of second and fourth powers of the R-charges. ${ }^{14}$ Taking again into account that the chiral anomaly receives only the contributions of the two opposite chirality fermions in table 3 , we get

$$
\begin{aligned}
\sum r^{2} & =\sum_{k=0}^{p-1}\left(-\frac{p-1}{2}+k\right)^{2}-\sum_{k=0}^{p-3}\left(-\frac{p-3}{2}+k\right)^{2}=\frac{1}{2}(p-1)^{2} \\
\sum r^{4} & =\sum_{k=0}^{p-1}\left(-\frac{p-1}{2}+k\right)^{4}-\sum_{k=0}^{p-3}\left(-\frac{p-3}{2}+k\right)^{4}=\frac{1}{8}(p-1)^{4} .
\end{aligned}
$$

Including the normalization constants so that for $p=2$ we recover the vector multiplet values in table 2 , we get

$$
\mathrm{V}_{p}^{(1,0)}: \quad \alpha=-(p-1)^{4}, \quad \beta=-\frac{1}{2}(p-1)^{2} .
$$

As expected, for $p=1$ the expressions for the chiral anomalies in (3.2) and (3.4) are the same as for $S^{(1,0)}$ in table 2 up to an opposite overall sign.

Next, let us turn to the conformal anomalies. To compute the conformal anomaly a-coefficient corresponding to $\mathrm{V}_{p}^{(1,0)}$ we may use the general expression for a of a field associated to a representation $\left(\Delta ; h_{1}, h_{2}, h_{3}\right)$ of the conformal group $\mathrm{SO}(2,6)$ given in $[8$, $43]: 15$

$$
\begin{aligned}
\mathrm{a}(\Delta ; \mathbf{h})=-\frac{(-1)^{2 \bar{h}} \mathrm{~d}(\mathbf{h})}{96} \times 37800 & (\Delta-3)\left[15(\Delta-3)^{6}\right. \\
& -21(\Delta-3)^{4}\left[h_{3}^{2}+h_{1}\left(h_{1}+4\right)+h_{2}\left(h_{2}+2\right)+5\right] \\
+ & 35(\Delta-3)^{2}\left[\left(h_{1}+2\right)^{2}\left(h_{2}+1\right)^{2}+\left(h_{1}\left(h_{1}+4\right)+h_{2}\left(h_{2}+2\right)+5\right) h_{3}^{2}\right] \\
- & \left.105\left(h_{1}+2\right)^{2}\left(h_{2}+1\right)^{2} h_{3}^{2}\right] .
\end{aligned}
$$

\footnotetext{
${ }^{14}$ Here we consider the Cartan $\mathrm{U}(1)$ subgroup of the $\mathrm{SU}(2)_{R}$ as the 4 th order anomaly $c_{2}^{2}$ is determined by the sum of fourth powers of the $J_{z}$ eigenvalue, see, e.g., [42].

${ }^{15}$ Here $\mathbf{h}=\left(h_{1}, h_{2}, h_{3}\right), \bar{h}=h_{1}+h_{2}+h_{3}$ and the dimension $\mathrm{d}(\mathbf{h})$ of the $\mathrm{SO}(6)$ representation $\mathbf{h}$ is $\mathrm{d}(\mathbf{h})=\frac{1}{12}\left(1+h_{1}-h_{2}\right)\left(1+h_{2}-h_{3}\right)\left(1+h_{2}+h_{3}\right)\left(2+h_{1}-h_{3}\right)\left(2+h_{1}+h_{3}\right)\left(2+h_{1}+h_{2}\right)$.

Note that

$\frac{\partial \mathrm{a}(\Delta ; \mathbf{h})}{\partial \Delta}=\frac{(-1)^{2 \bar{h}} \mathrm{~d}(\mathbf{h})}{96 \times 360}\left(\Delta-h_{1}+5\right)\left(\Delta+h_{1}-1\right)\left(\Delta-h_{2}-4\right)\left(\Delta+h_{2}-2\right)\left(\Delta-h_{3}-3\right)\left(\Delta+h_{3}-3\right)$.
} 
Summing up contributions of all fields with their multiplicities in table 3 , we get ${ }^{16}$

$$
\mathrm{V}_{p}^{(1,0)}: \quad \mathrm{a}=\frac{1}{72}(p-1)^{4}-\frac{1}{144}(p-1)^{2}+\frac{11}{34560} .
$$

For $p=2$ this gives the value for $V^{(1,0)}$ in $(2.3) .{ }^{17}$

It is straightforward also to directly compute the conformal anomaly on a Ricci flat background by assuming that Weyl-covariant kinetic operators appearing in the generalization of the Lagrangian (3.1) to curved background factorize into a product of the standard 2-derivative operators (cf. appendix A). We then find

$$
\begin{aligned}
\mathrm{V}_{p}^{(1,0)}:\left.A_{6}\right|_{R_{m n}=0}= & {[(p+1)(2 p-3)+(p-3)(2 p-1)] A_{6}(\varphi)+(p-1)(2 p-2) A_{6}(V) } \\
& +[p(4 p-5)+(p-2)(4 p-3)] A_{6}(\psi)=-\frac{1}{11520} E_{6} .
\end{aligned}
$$

Here $A_{6}(\varphi), A_{6}(V)$ and $A_{6}(\psi)$ are conformal anomalies for standard Laplacians defined on scalars, vectors and spinors on a Ricci flat background. This expression was derived for $p>2$ but is also valid for $p=2$, i.e. for $V^{(1,0)}$ when the vector is a gauge-invariant one.

Comparing (3.7) to the general expression in (1.6) and using the expression for a in (3.6) we conclude that in addition to the expected relation (1.7), i.e. $c_{3}=\frac{1}{6}\left(c_{1}-2 c_{2}\right)$, we get

$$
\mathrm{V}_{p}^{(1,0)}: \quad \mathrm{c}_{1}+4 \mathrm{c}_{2}=192 \mathrm{a}-\frac{1}{60}=\frac{8}{3}(p-1)^{4}-\frac{4}{3}(p-1)^{4}+\frac{2}{45} .
$$

Using the results for the chiral anomalies in (3.2), (3.4) we conclude that the value of the a-coefficient in (3.6) is indeed consistent with the general expression (1.13) of [1]. Our suggested expressions (1.15) for $\mathrm{c}_{1}$ and $\mathrm{c}_{2}$ give

$$
\mathrm{c}_{1}=\frac{4}{3}(p-1)^{4}-\frac{2}{3}(p-1)^{2}+\frac{1}{27}, \quad \mathrm{c}_{2}=\frac{1}{3}(p-1)^{4}-\frac{1}{6}(p-1)^{2}+\frac{1}{540} .
$$

\footnotetext{
${ }^{16}$ Explicitly, the separate contributions a $\left(\Delta ; h_{1}, h_{2}, h_{3}\right)$ are

$\mathrm{a}(2 p ; 0,0,0)=-\frac{1}{1890}(p-1)^{7}+\frac{1}{540}(p-1)^{6}-\frac{1}{540}(p-1)^{5}+\frac{11}{12960}(p-1)^{3}-\frac{1}{2880}(p-1)^{2}+\frac{1}{72576}$,

$\mathrm{a}\left(2 p+\frac{1}{2} ; \frac{1}{2}, \frac{1}{2},-\frac{1}{2}\right)=\frac{2}{945}(p-1)^{7}-\frac{1}{270}(p-1)^{6}-\frac{1}{270}(p-1)^{5}+\frac{1}{144}(p-1)^{4}+\frac{1}{810}(p-1)^{3}$$$
-\frac{1}{360}(p-1)^{2}+\frac{191}{1451520},
$$$$
\mathrm{a}(2 p+1 ; 1,0,0)=-\frac{1}{315}(p-1)^{7}+\frac{1}{90}(p-1)^{5}-\frac{1}{240}(p-1)^{3},
$$$$
\mathrm{a}\left(2 p+\frac{3}{2} ; \frac{1}{2}, \frac{1}{2}, \frac{1}{2}\right)=\frac{2}{945}(p-1)^{7}+\frac{1}{270}(p-1)^{6}-\frac{1}{270}(p-1)^{5}-\frac{1}{144}(p-1)^{4}+\frac{1}{810}(p-1)^{3}
$$$$
+\frac{1}{360}(p-1)^{2}-\frac{191}{1451520},
$$$$
\mathrm{a}(2 p+2 ; 0,0,0)=-\frac{1}{1890}(p-1)^{7}-\frac{1}{540}(p-1)^{6}-\frac{1}{540}(p-1)^{5}+\frac{11}{12960}(p-1)^{3}+\frac{1}{2880}(p-1)^{2}-\frac{1}{72576} .
$$

${ }^{17}$ The expression (3.6) was derived assuming $p>1$; still, if we formally set $p=1$ we get the value opposite to the value of $S^{(1,0)}$ in table 1 while the conformal anomaly of $\mathrm{V}_{1}^{(1,0)}=S^{(0,1)}$ (cf. table 3) should be the same as of $S^{(1,0)}$.
} 


\begin{tabular}{|c|c|c|c|c|c|}
\hline & $\left(\Delta ; h_{1}, h_{2}, h_{3}\right)$ & $\mathrm{USp}(4)$ & & $\left(\Delta ; h_{1}, h_{2}, h_{3}\right)$ & $\mathrm{USp}(4)$ \\
\hline \multirow{6}{*}{$p \geq 2$} & $(2 p ; 0,0,0)$ & {$[0, p]$} & \multirow{10}{*}{$p \geq 4$} & \multirow{10}{*}{$\begin{array}{l}(2 p+2 ; 0,0,0) \\
\left(2 p+\frac{5}{2} ; \frac{1}{2}, \frac{1}{2}, \frac{1}{2}\right) \\
(2 p+3 ; 1,0,0) \\
\left(2 p+\frac{7}{2} ; \frac{1}{2}, \frac{1}{2},-\frac{1}{2}\right) \\
(2 p+4 ; 0,0,0)\end{array}$} & \multirow{10}{*}{$\begin{array}{l}{[4, p-4]} \\
{[3, p-4]} \\
{[2, p-4]} \\
{[1, p-4]} \\
{[0, p-4]}\end{array}$} \\
\hline & $\left(2 p+\frac{1}{2} ; \frac{1}{2}, \frac{1}{2}, \frac{1}{2}\right)$ & {$[1, p-1]$} & & & \\
\hline & $(2 p+1 ; 1,1,1)$ & {$[0, p-1]$} & & & \\
\hline & $(2 p+1 ; 1,0,0)$ & {$[2, p-2]$} & & & \\
\hline & $\left(2 p+\frac{3}{2} ; \frac{3}{2}, \frac{1}{2}, \frac{1}{2}\right)$ & {$[1, p-2]$} & & & \\
\hline & $(2 p+2 ; 2,0,0)$ & {$[0, p-2]$} & & & \\
\hline \multirow{4}{*}{$p \geq 3$} & $\left(2 p+\frac{3}{2} ; \frac{1}{2}, \frac{1}{2},-\frac{1}{2}\right)$ & {$[3, p-3]$} & & & \\
\hline & $(2 p+2 ; 1,1,0)$ & {$[2, p-3]$} & & & \\
\hline & $\left(2 p+\frac{5}{2} \frac{3}{2}, \frac{1}{2},-\frac{1}{2}\right)$ & {$[1, p-3]$} & & & \\
\hline & $(2 p+3 ; 1,1,-1)$ & {$[0, p-3]$} & & & \\
\hline
\end{tabular}

Table 4. $\mathrm{SO}(2,6) \times \mathrm{USp}(4)$ representations of fields of $11 \mathrm{~d}$ supergravity on $\mathrm{AdS}_{7} \times S^{4}$ vacuum. Each level $p$ corresponds to $6 \mathrm{~d}(2,0)$ superconformal multiplet. The canonical dimension of the corresponding $6 \mathrm{~d}$ fields is $\Delta_{-}=6-\Delta$.

These are indeed consistent with (3.8), thus providing an additional test of eq. (1.15). Let us note also that $\mathrm{c}_{1}-4 \mathrm{c}_{2}=\frac{4}{135}$ is independent of $p$, i.e. is the same as for $V^{(1,0)} \cdot{ }^{18}$

\section{Higher derivative spin 2 superconformal multiplets}

In this section we shall consider the chiral and conformal anomalies of higher derivative $6 \mathrm{~d}$ superconformal multiplets generalizing $(2,0)$ conformal supergravity (CSG) multiplet.

Let us start with the Kaluza-Klein spectrum [44-46] of 11d supergravity compactified on $S^{4}$ given in table 4 (see also [47]). The massless level $p=2$ is represented by the fields of maximal gauged $7 \mathrm{~d}$ supergravity with $\mathrm{AdS}_{7}$ vacuum. The corresponding 6d "massless" $\operatorname{OSp}(2,6 \mid 4)$ superconformal multiplet is that of $(2,0)$ conformal supergravity $[8,48]{ }^{19}$ Similarly, the $7 \mathrm{~d}$ multiplet formed by fields belonging to $p>2$ level corresponds to higher derivative "massive" $(2,0)$ superconformal multiplet in $6 \mathrm{~d}$ (which we shall denote as $\left.\mathrm{CSG}_{p}\right) .{ }^{20}$ Equivalently, just like the higher derivative vector multiplets in table 3 appear in the product of $p$ copies of $(1,0)$ hypermultiplet $S^{(1,0)}$, the $\mathrm{CSG}_{p}$ multiplets appear in the product of $p$ copies of $(2,0)$ tensor multiplet $T^{(2,0)}[45]$.

\footnotetext{
${ }^{18}$ Once again, for $p=1$ the $\mathrm{c}_{i}$ in $(3.9)$ are opposite to the values corresponding to $S^{(1,0)}$ in table 1 which is due to the fact that we applied the relations between chiral and conformal anomalies valid for $(1,0)$ multiplets while the $p=1$ example is formally a $(0,1)$ scalar multiplet (cf. footnote above eq. (1.13)).

${ }^{19}$ In table 4 we have chosen chirality assignments so that for $p=2$ they correspond to the canonical choice in the $(2,0)$ conformal supergravity [48], i.e. the fermions and gravitino have positive chirality and the antisymmetric tensor is self-dual.

${ }^{20}$ Fields of "massive" conformal group representations do not have gauge invariances that are present in the "massless" case.
} 
Below we shall compute the chiral (gravitational and R-symmetry) anomalies and the conformal anomaly c-coefficient in $(1.8)$ of these $(2,0) \mathrm{CSG}_{p}$ multiplets complementing the result for their conformal anomaly a-coefficient found in $[8,43]$. The anomaly coefficients will all be proportional to the same factor $6 p(p-1)+1$ and will satisfy the expected relations (1.17), (1.19), (1.21). In particular, we will confirm the conjecture of [8] that the system of $(2,0)$ conformal supergravity coupled to 26 tensor multiplets is completely anomaly free.

\subsection{Chiral anomaly coefficients}

The fields in table 4 contributing to chiral (gravitational and R-symmetry) anomalies in (1.11) are the MW fermions $\psi^{ \pm} \sim\left(\frac{1}{2}, \frac{1}{2}, \pm \frac{1}{2}\right)$, MW conformal gravitini $\psi_{m}^{ \pm} \sim\left(\frac{3}{2}, \frac{1}{2}, \pm \frac{1}{2}\right)$ and (anti)self-dual rank 3 antisymmetric tensors $A_{m n k}^{ \pm} \sim(1,1, \pm 1)$. Contributions of these fields are to be summed up with multiplicities corresponding to their $\mathrm{USp}(4)=\mathrm{SO}(5)$ R-symmetry representations. ${ }^{21}$

To find USp(4) multiplicity of a particular conformal field at level $p$ one is to add up dimensions of the corresponding USp(4) representations in table 4. Since the positivechirality fields contribute to chiral anomalies with the opposite sign compared to the negative-chirality ones, we may express the total result in terms of the effective numbers of, e.g., the positive-chirality fields. Counting the negative chirality fermions as minus the positive chirality ones we find for the effective number of the positive chirality MW spinors $\psi^{+} \sim\left(\frac{1}{2}, \frac{1}{2}, \frac{1}{2}\right)$ at level $p$ :

$$
n\left(\psi^{+}\right)=\operatorname{dim}(1, p-1)-\operatorname{dim}(3, p-3)+\operatorname{dim}(3, p-4)-\operatorname{dim}(1, p-4)=2 p(p-1)+8 .
$$

Similarly, for the effective number of positive chirality MW gravitini we find $n\left(\psi_{m}^{+}\right)=$ $2 p(p-1)$, while the effective number of self-dual 3-form fields is $n\left(A_{m n k}^{+}\right)=2 p(p-1)+1$.

The conformal $6 \mathrm{~d}$ fields corresponding to representations in table 4 are non-standard having higher-derivative kinetic terms (with the number of derivatives determined by canonical dimension $6-\Delta$ ). In general, the chiral anomalies of higher-derivative fermions will be same as anomalies of their lowest-derivative counterparts. For the gravitino $\psi_{m}^{+}$and the antisymmetric tensor $A_{m n k}^{+}$at levels $p>2$ we will have an additional complication: they will be conformal and "massive" (i.e. will not have usual gauge invariance).

Let us start with the gravitational anomalies and first recall the expressions [24] for the purely gravitational parts of the $6 \mathrm{~d}$ anomaly polynomial $\mathcal{I}_{8}$ in $(1.11)$ for the positive chirality MW fermion, the standard gauge-invariant (real) self-dual rank 3 antisymmetric tensor $\left(A \equiv H=d T\right.$, with potential denoted as $\left.T^{+}\right)$and the standard (1st-derivative,

\footnotetext{
${ }^{21}$ The dimension of the $\operatorname{USp}(4)$ representation $[a, b](a, b$ are Dynkin labels) is $\operatorname{dim}(a, b)=\frac{1}{6}(a+1)(b+1)(a+b+2)(a+2 b+3)$.
} 
gauge-invariant) positive chirality MW gravitino ${ }^{22}$

$$
\begin{array}{ll}
\mathcal{I}_{8}\left(\frac{1}{2}^{+}\right)=-\frac{1}{16 \times 6 !}\left(7 \mathrm{p}_{1}^{2}-4 \mathrm{p}_{2}\right), & \mathcal{I}_{8}\left(T^{+}\right)=-\frac{1}{16 \times 6 !}\left(32 \mathrm{p}_{1}^{2}-224 \mathrm{p}_{2}\right), \\
\mathcal{I}_{8}\left(\frac{3}{2}^{+}\right)=-\frac{1}{16 \times 6 !}\left(275 \mathrm{p}_{1}^{2}-980 \mathrm{p}_{2}\right) .
\end{array}
$$

Given a generic rank 3 antisymmetric tensor (with $20=\left(\begin{array}{l}6 \\ 3\end{array}\right)$ components) we may represent it in terms of two independent transverse 2 -tensors $T_{m n}, \widetilde{T}_{m n}$ with $10+10$ components as $^{23}$

$$
A_{m n k}=\partial_{[m} T_{n k]}+\epsilon_{m n k l p q} \partial_{[l} \widetilde{T}_{p q]} \cdot
$$

Here only the transverse parts of $T$ and $\tilde{T}$ contribute, i.e. $A$ is thus expressed in terms of two standard gauge-invariant 3-form field strengths $H=d T$ and $\widetilde{H}=d \widetilde{T}$. Similarly, the contribution of a self-dual $A$ to chiral anomalies will be equivalent to the contributions of self-dual parts of $H$ and $\widetilde{H}$, i.e. it will be twice the standard antisymmetric tensor contribution in (4.1) (or, equivalently, the contribution of one complex $T^{+}$field).

The gravitino $\psi_{m}^{ \pm} \sim\left(\frac{3}{2}, \frac{1}{2}, \pm \frac{1}{2}\right)$ in table 4 is different from the standard gravitino discussed in [24] in two respects (in addition to having higher-derivative kinetic term): (i) for any $p$ it is conformal (i.e. its $\gamma$-trace is zero); (ii) for $p>2$ it has no gauge invariance. Thus compared to the standard negative-chirality gravitino anomaly $\mathcal{I}_{8}\left(\frac{3}{2}^{+}\right)$in (4.1) the anomaly of the massive conformal $\psi_{m}^{+}$should have the negative-chirality fermion ( $\gamma$-trace) anomaly $\mathcal{I}_{8}\left(\frac{1}{2}^{-}\right)=-\mathcal{I}_{8}\left(\frac{1}{2}^{+}\right)$subtracted and the positive-chirality fermion $\left(\partial_{m} \epsilon\right.$ gauge degree of freedom) anomaly $\mathcal{I}_{8}\left(\frac{1}{2}^{+}\right)$added, or, equivalently, the ghost contribution should not be subtracted. Thus for $p \geq 2$ we should have

$$
\mathcal{I}_{8}\left(\psi_{m}^{+}\right)=\mathcal{I}_{8}\left(\frac{3}{2}^{+}\right)+2 \mathcal{I}_{8}\left(\frac{1}{2}^{+}\right) .
$$

As a result, the total gravitational anomaly polynomial of $\mathrm{CSG}_{p}$ multiplet is found to be

$$
\begin{aligned}
\mathcal{I}_{8}\left(\mathrm{CSG}_{p}\right)=[ & 2 p(p-1)+8] \mathcal{I}_{8}\left(\frac{1}{2}^{+}\right)+2[2 p(p-1)+1] \mathcal{I}_{8}\left(T^{+}\right) \\
& +2 p(p-1)\left[\mathcal{I}_{8}\left(\frac{3}{2}^{-}\right)+2 \mathcal{I}_{8}\left(\frac{1}{2}^{+}\right)\right] \\
=- & \frac{1}{96}[6 p(p-1)+1]\left(\mathrm{p}_{1}^{2}-4 \mathrm{p}_{2}\right) .
\end{aligned}
$$

The corresponding gravitational anomaly coefficients in (1.11) are thus

$$
\mathrm{CSG}_{p}: \quad \gamma=-\frac{1}{4} \delta=-\frac{1}{4}[6 p(p-1)+1] .
$$

This is in agreement with the relation (1.17) expected for $(2,0)$ multiplets.

\footnotetext{
${ }^{22}$ Using the field content $(2.1),(2.2)$ of the $(1,0)$ scalar, tensor and vector multiplets one can check that the values of $\gamma, \delta$ in table 2 indeed follow from (4.1).

${ }^{23}$ This is analogous to the representation of the antisymmetric rank 2 tensors in $4 \mathrm{~d}$ conformal supergravity used in section 2.3 of [49], see also a discussion in [30].
} 
The expressions (4.4), (4.5) were derived for $p>2$ (when gravitino is massive) but are actually valid also for $p=2$ : in this case the gauge-invariant conformal gravitino contribution is $\mathcal{I}_{8}\left(\frac{3}{2}{ }^{+}\right)+\mathcal{I}_{8}\left(\frac{1}{2}^{+}\right)$but we formally get also "extra" 4 chiral fermion contributions from the multiplicity factor (see appendix B.2).

Similar results are found for the R-symmetry and mixed anomaly coefficients $\alpha$ and $\beta$ in (1.11) (see appendix B):

$$
\mathrm{CSG}_{p}: \quad \alpha=2 \beta=-2[6 p(p-1)+1] .
$$

We thus observe that for any $p$ all 4 chiral anomaly coefficients $\vec{\alpha}=(\alpha, \beta, \gamma, \delta)$ are proportional to the chiral anomaly coefficients of the $T^{(2,0)}$ multiplet in table 2 , i.e.

$$
\vec{\alpha}\left(\mathrm{CSG}_{p}\right)=-2[6 p(p-1)+1] \vec{\alpha}\left(T^{(2,0)}\right), \quad \vec{\alpha}\left(T^{(2,0)}\right)=\left(1, \frac{1}{2}, \frac{1}{8},-\frac{1}{2}\right) .
$$

In particular, for $p=2$ when $\mathrm{CSG}_{p}$ is the multiplet of $(2,0)$ conformal supergravity, we get

$$
\vec{\alpha}\left(\mathrm{CSG}^{(2,0)}\right)+26 \vec{\alpha}\left(T^{(2,0)}\right)=0 .
$$

As a result, the gravitational and R-symmetry anomalies of $(2,0)$ conformal supergravity can be cancelled by adding 26 tensor multiplets $T^{(2,0)}$.

\subsection{Conformal anomaly coefficients}

The conformal anomaly a-coefficient for $\mathrm{CSG}_{p}$ multiplet was found in $[8,43]$ by a computation on $S^{6}$

$$
\mathrm{a}\left(\mathrm{CSG}_{p}\right)=-2[6 p(p-1)+1] \mathrm{a}\left(T^{(2,0)}\right), \quad \mathrm{a}\left(T^{(2,0)}\right)=-\frac{7}{1152},
$$

where $\mathrm{a}\left(T^{(2,0)}\right)$ is the $(2,0)$ tensor multiplet value in (1.14). Comparing the expression (4.9) with the chiral anomaly result $(4.7)$ we conclude that the $(1,0)$ relation $(1.13)$ of $[1]$ or the relation for a-coefficient in (1.19) is indeed satisfied.

While a direct computation of the $c_{i}$ anomaly coefficients in (1.4) for the $\mathrm{CSG}_{p}$ multiplet may be feasible by assuming factorization of all kinetic operators on a Ricci flat background, here we shall apply the expected relations (1.15) or (1.19) between chiral and conformal anomalies of superconformal multiplets implying in view of (4.7) that $c_{i}$ in (1.4) are all proportional to $\mathrm{c}$ as in (1.8) where $\mathrm{c}$ is given by (1.19), (1.21)

$$
\mathrm{c}=\mathrm{a}-\frac{1}{192} \delta=-2[6 p(p-1)+1] \mathrm{c}\left(T^{(2,0)}\right), \quad \mathrm{c}\left(T^{2,0}\right)=-\frac{1}{288} .
$$

As a result, applying this to the $p=2$ case we conclude that all chiral and conformal anomalies of $(2,0)$ system of $6 \mathrm{~d}$ conformal supergravity plus 26 tensor multiplets vanish.

\section{Some interacting $(1,0)$ superconformal theories}

Let us now consider some examples of interacting $(1,0)$ superconformal theories. 


\begin{tabular}{|c|c|c|c|c|}
\hline & $\alpha$ & $\beta$ & $\gamma$ & $\delta$ \\
\hline $\mathcal{E}_{N}$ & $N\left(4 N^{2}+6 N+3\right)$ & $-\frac{N}{2}(6 N+5)$ & $\frac{7 N}{8}$ & $-\frac{N}{2}$ \\
\hline $\mathcal{T}_{N, \Gamma}$ & $|\Gamma|^{2} N^{3}-2 N|\Gamma|\left(r_{\Gamma}+1\right)$ & $N-\frac{N}{2}|\Gamma|\left(r_{\Gamma}+1\right)+\frac{d_{\Gamma}}{2}$ & $\frac{N}{8}+\frac{7 d_{\Gamma}}{240}$ & $-\frac{N}{2}-\frac{d_{\Gamma}}{60}$ \\
& $+2 N+d_{\Gamma}$ & & & \\
\hline
\end{tabular}

Table 5. Chiral anomaly coefficients for the $\mathcal{E}_{N}$ and $\mathcal{T}_{N, \Gamma}(1,0)$ theories.

First, let us summarize the expressions for the chiral, gravitational [50, 51], and conformal $[19,43,52-54]$ anomaly coefficients of interacting $(2,0) A_{N}$ theory describing $N$ coincident M5 branes:

$$
\begin{aligned}
& (2,0): \quad \alpha=N^{3}-1, \quad \beta=4 \gamma=-\delta=\frac{1}{2}(N-1), \\
& \mathrm{a}=-\frac{1}{288}\left(4 N^{3}-\frac{9}{4} N-\frac{7}{4}\right)=-\frac{1}{288}(N-1)\left[(2 N+1)^{2}+\frac{3}{4}\right], \\
& \mathrm{c}=-\frac{1}{288}\left(4 N^{3}-3 N-1\right)=-\frac{1}{288}(N-1)(2 N+1)^{2}, \quad \mathrm{c}_{1}=4 \mathrm{c}_{2}=-12 \mathrm{c}_{3}=96 \mathrm{c} .
\end{aligned}
$$

These values are perfectly consistent with (1.17)-(1.21). The leading order $N^{3}$ terms in (5.2), (5.3) follow [19] from $7 \mathrm{~d}$ supergravity $(R+\Lambda)$ terms found upon compactification of $11 \mathrm{~d}$ theory on $S^{4}$, the subleading order $N$ terms originate from $R^{4}$ corrections in $11 \mathrm{~d}$ action [52] and order $N^{0}$ terms are reproduced by 1-loop 11d supergravity corrections [43, $55]$.

There are also two cases of interacting $(1,0)$ superconformal theories with known chiral anomalies. The first is the $\mathcal{E}_{N}$ theory [56] on the world-volume of $N$ small coincident $E_{8}$ instantons in the heterotic string (E-string), or, equivalently, the theory on $N$ M5-branes on Horava-Witten $E_{8} 9$-brane. The second is the $\mathcal{T}_{N, \Gamma}$ theory describing $N$ M5-branes on the orbifold singularity $\mathbb{C}^{2} / \Gamma$ where $\Gamma$ is a discrete subgroup of $\operatorname{SU}(2)$ (see $[54,57]$ ). The corresponding coefficients in the anomaly polynomial (1.11) are given in table 5. Here $|\Gamma|$ is the order of the discrete group $\Gamma$ and $r_{\Gamma}$ and $d_{\Gamma}$ are the rank and dimension of the associated Lie algebra $G_{\Gamma}$ (e.g., $G_{\Gamma}=\mathrm{SU}(k)$ for $\Gamma=\mathbb{Z}_{k}$ ).

It follows from (1.14) that the corresponding a-anomaly coefficients are [1]

$$
\begin{aligned}
\mathrm{a}\left(\mathcal{E}_{N}\right) & =\frac{16}{7}\left(4 N^{3}+9 N^{2}+\frac{99}{16} N\right) \mathrm{a}_{\mathrm{T}}, \\
\mathrm{a}\left(\mathcal{T}_{N, \Gamma}\right) & =\frac{16}{7}\left(|\Gamma|^{2} N^{3}-\frac{3}{2}\left[|\Gamma|\left(r_{\Gamma}+1\right)-\frac{5}{8}\right] N+\frac{251}{480} d_{\Gamma}\right) \mathrm{a}_{\mathrm{T}} .
\end{aligned}
$$

From the expressions (1.15) with the chiral anomaly coefficients from table 5 we find

$$
\begin{aligned}
\mathrm{c}_{1}\left(\mathcal{E}_{N}\right) & =-\frac{16}{3} N^{3}-12 N^{2}-\frac{76}{9} N, \quad \mathrm{c}_{2}\left(\mathcal{E}_{N}\right)=-\frac{4}{3} N^{3}-3 N^{2}-\frac{17}{9} N, \\
\mathrm{c}_{1}\left(\mathcal{T}_{N, \Gamma}\right) & =-\frac{4}{3}|\Gamma|^{2} N^{3}+\left[2|\Gamma|\left(r_{\Gamma}+1\right)-1\right] N-\frac{19}{27} d_{\Gamma},
\end{aligned}
$$




$$
\mathrm{c}_{2}\left(\mathcal{T}_{N, \Gamma}\right)=-\frac{1}{3}|\Gamma|^{2} N^{3}+\frac{1}{4}\left[2|\Gamma|\left(r_{\Gamma}+1\right)-1\right] N-\frac{91}{540} d_{\Gamma} .
$$

Here the leading large $N$ terms $\left(\sim N^{3}\right)$ in $c_{1}$ and $c_{2}$ are in ratio $4: 1$ as for a $(2,0)$ theory (cf. (1.8)). This is what should be expected from the AdS/CFT as the $N^{3}$ terms originate from the universal Einstein term in the dual $7 \mathrm{~d}$ supergravity action.

Furthermore, a similar relation is true also for the first subleading terms, $\mathcal{O}\left(N^{2}\right)$ in $\mathcal{E}_{N}$ case and $\mathcal{O}(N)$ in $\mathcal{T}_{N, \Gamma}$ case, i.e.

$$
\begin{gathered}
\mathrm{c}_{1}\left(\mathcal{E}_{N}\right)-4 \mathrm{c}_{2}\left(\mathcal{E}_{N}\right)=0 \cdot N^{3}+0 \cdot N^{2}-\frac{8}{9} N, \\
\mathrm{c}_{1}\left(\mathcal{T}_{N, \Gamma}\right)-4 \mathrm{c}_{2}\left(\mathcal{T}_{N, \Gamma}\right)=0 \cdot N^{3}+0 \cdot N-\frac{4}{135} d_{\Gamma} .
\end{gathered}
$$

This fact should also have a holographic explanation, cf. [58], which may thus provide an independent check of the relations (1.15).

Another indirect support for our relations (1.15) comes from consideration of a class of $6 \mathrm{~d}(1,0)$ theories named "very Higgsable" in [59]: they admit a completely Higgsed branch where no tensor multiplets remain. Compactifying these theories on $T^{2}$ one gets $4 \mathrm{~d} \mathcal{N}=2$ superconformal theories with the $4 \mathrm{~d}$ conformal anomaly coefficients $\left(\mathrm{a}_{4 \mathrm{~d}}, \mathrm{c}_{4 \mathrm{~d}}\right)$ determined in terms of the coefficients in the $6 \mathrm{~d}$ anomaly polynomial $\mathcal{I}_{8}$ in (1.11) as [59]. In our notations,

$$
\mathrm{a}_{4 \mathrm{~d}}=\frac{1}{4 !}(-12 \beta+24 \gamma-18 \delta), \quad \mathrm{c}_{4 \mathrm{~d}}=\frac{1}{4 !}(-12 \beta+64 \gamma-8 \delta) .
$$

Using (5.9) together with (1.15) we then find the following remarkable identity

$$
\mathrm{c}_{4 \mathrm{~d}}-\mathrm{a}_{4 \mathrm{~d}}=-\frac{45}{32}\left(\mathrm{c}_{1}-4 \mathrm{c}_{2}\right) .
$$

This relates the combinations of the conformal anomaly coefficients that vanish in the maximally supersymmetric cases, i.e. $\mathcal{N}=4$ in $4 \mathrm{~d}$ and $(2,0)$ in $6 \mathrm{~d}$.

\section{6d conformal anomaly from $7 \mathrm{~d}$ gravitational effective action}

As already mentioned above, an important source of information about conformal anomalies of interacting $6 \mathrm{~d}$ superconformal theories is AdS/CFT [19, 52]. One may expect that strong-coupling limit of a $6 \mathrm{~d}$ superconformal theory is described by some effective locally supersymmetric $7 \mathrm{~d}$ theory with an $\mathrm{AdS}_{7}$ vacuum.

Such 7d action may arise, e.g., from 11d M-theory effective action upon compactification on some 4 -space $\left(S^{4}\right.$ in the case of the standard $(2,0)$ theory). Ignoring for simplicity all other fields than the metric, the effective $7 \mathrm{~d}$ Lagrangian will have the form $\mathcal{L}=R+\Lambda+R^{2}+R^{3}+R^{4}+\ldots$ where the expansion in powers of curvature should correspond to the strong-coupling (large $N$ ) expansion in the boundary $6 \mathrm{~d}$ theory. The combinations of the curvature invariants should be such that they admit supersymmetrization consistent with the amount of supersymmetry of the boundary theory. That may provide constraints $(1.7),(1.8)$ on the conformal anomaly $c_{i}$-coefficients [15] that we shall discuss below. 


\subsection{Quadratic and cubic curvature corrections: linearized approximation}

Let us consider the $7 \mathrm{~d}$ action including the most general quadratic and cubic curvature invariants $^{24}$

$$
\begin{aligned}
S & =-\frac{1}{2 \kappa_{7}^{2}} \int d^{7} x \sqrt{-g}\left(R+\frac{30}{L^{2}}+\Delta \mathcal{L}\right), \\
\Delta \mathcal{L} & =L^{2} \sum_{i=1}^{3} u_{2, i} I_{2, i}+L^{4} \sum_{i=1}^{8} u_{3, i} I_{3, i} .
\end{aligned}
$$

Here $L$ is a scale (which to leading order is the same as the $\mathrm{AdS}_{7}$ radius) introduced to make the coefficients $u_{n, i}$ dimensionless and $I_{r, i}$ are curvature contractions

$$
\begin{array}{rlrl}
I_{2,1}=R_{\mu \nu \lambda \rho}^{2}, & I_{2,2}=R_{\mu \nu}^{2}, & I_{2,3}=R^{2}, \\
I_{3,1}=R^{\mu \nu \lambda \rho} R_{\rho \rho \kappa \alpha} R^{\kappa \alpha}{ }_{\mu \nu}, I_{3,2} & =R^{\mu \nu}{ }_{\lambda \rho} R^{\lambda \kappa}{ }_{\nu \alpha} R^{\rho \alpha}{ }_{\mu \kappa}, & I_{3,3}=R^{\mu \lambda \rho \kappa} R_{\rho \kappa \lambda \alpha} R^{\alpha}{ }_{\mu}, \\
I_{3,4}=R R_{\mu \nu \lambda \rho}^{2}, & I_{3,5} & =R^{\mu \nu \rho \lambda} R_{\rho \mu} R_{\lambda \nu}, & I_{3,6}=R^{\mu \nu} R_{\nu \lambda} R^{\lambda}{ }_{\mu}, \\
I_{3,7}=R R_{\mu \nu}^{2}, & I_{3,8} & =R^{3} .
\end{array}
$$

The first two terms in (6.1) can be embedded into the maximal $7 \mathrm{~d}$ gauged supergravity so should describe the $(2,0)$ superconformal theory at the boundary, while the higher order terms may or may not (partially) break supersymmetry.

Starting with (6.1), one may compute the coefficients of the boundary conformal anomaly by generalizing the approach of [19], i.e. extracting the IR logarithmic singularity in the action evaluated on a classical solution with prescribed metric at the boundary. Keeping only terms linear in coefficients $u_{r, k}$ in $(6.2)^{25}$ one finds for $c_{i}$ in $(1.4)[15,31]^{26}$

$$
\begin{aligned}
\mathrm{c}_{1}=\mathrm{k} & {\left[-96+3072\left(\frac{5}{96} u_{2,1}+\frac{21}{32} u_{2,2}+\frac{147}{32} u_{2,3}\right.\right.} \\
& \left.\left.+\frac{9}{16} u_{3,1}+\frac{23}{16} u_{3,2}+\frac{5}{16} u_{3,3}-\frac{35}{16} u_{3,4}-\frac{63}{16} u_{3,5}-\frac{63}{16} u_{3,6}-\frac{441}{16} u_{3,7}-\frac{3087}{16} u_{3,8}\right)+\ldots\right], \\
\mathrm{c}_{2}=\mathrm{k} & {\left[-24+3072\left(\frac{37}{384} u_{2,1}+\frac{21}{128} u_{2,2}+\frac{147}{128} u_{2,3}\right.\right.} \\
& \left.\left.+\frac{9}{64} u_{3,1}+\frac{7}{64} u_{3,2}+\frac{37}{64} u_{3,3}-\frac{259}{64} u_{3,4}-\frac{63}{64} u_{3,5}-\frac{63}{64} u_{3,6}-\frac{441}{64} u_{3,7}-\frac{3087}{64} u_{3,8}\right)+\ldots\right], \\
\mathrm{c}_{3}= & \mathrm{k}\left[8+3072\left(\frac{3}{128} u_{2,1}-\frac{7}{128} u_{2,2}-\frac{49}{128} u_{2,3}\right.\right. \\
& \left.\left.-\frac{41}{192} u_{3,1}-\frac{31}{192} u_{3,2}+\frac{9}{64} u_{3,3}-\frac{63}{64} u_{3,4}+\frac{21}{64} u_{3,5}+\frac{21}{64} u_{3,6}+\frac{147}{64} u_{3,7}+\frac{1029}{64} u_{3,8}\right)+\ldots\right] .
\end{aligned}
$$

\footnotetext{
${ }^{24}$ We shall ignore terms with derivatives of the curvature as they will not contribute to the relevant conformal anomaly coefficients.

${ }^{25}$ Non-linear corrections in the case of special $R^{2}$ and $R^{3}$ combinations representing Euler densities $E_{4}$ and $E_{6}$ were computed in [31, 60-62], see section 6.2 below.

${ }^{26}$ We thank M. Kulahizi and A. Parnachev for sending us a corrected version of the list of coefficients in [15].
} 
Here dots stand for terms of higher order in $u_{r, i}$ and the dimensionless factor $\mathrm{k}$ is

$$
\mathrm{k}=\frac{\pi^{3} L^{5}}{48 \kappa_{7}^{2}}
$$

The leading order $\left(u_{r, i}=0\right)$ values [19] are those of the large $N$ limit of the $(2,0)$ theory for which

$$
\frac{L^{5}}{2 \kappa_{7}^{2}}=\frac{N^{3}}{3 \pi^{3}}, \quad \text { i.e. } \quad \mathrm{k}=\frac{N^{3}}{72},
$$

i.e. $\mathrm{c}_{i}$ are given by the $N^{3}$ terms in (5.3) with $\mathrm{c}=-\mathrm{k}=-\frac{N^{3}}{72}$ (which is $4 N^{3}$ times the $\mathrm{c}$ anomaly of one free $(2,0)$ tensor multiplet $[7])$.

To find the a-coefficient in (1.4) for the theory (6.1) one may follow the approach used in $4 \mathrm{~d}$ case in [63] and compute the gravitational effective action on the corrected $\mathrm{AdS}_{7}$ solution with the value of the radius that extremises the action evaluated on a test AdS solution. ${ }^{27}$ For the $\mathrm{AdS}_{7}$ metric with radius $r$ we have

$$
R_{\mu \nu \kappa \lambda}=-\frac{1}{r^{2}}\left(g_{\mu \kappa} g_{\nu \lambda}-g_{\mu \lambda} g_{\nu \kappa}\right), \quad R_{\mu \nu}=-\frac{6}{r^{2}} g_{\mu \nu}, \quad R=-\frac{42}{r^{2}}
$$

The a-anomaly is then proportional to the on-shell value of the action evaluated at the extremal value $r_{*}$ of the radius

$$
\begin{aligned}
\mathrm{a} & =-\mathrm{k} A\left(r_{*}\right), & A(r) & =-\frac{1}{12 L^{5}} r^{7}\left(-\frac{42}{r^{2}}+\frac{30}{L^{2}}\right)+O\left(u_{r, i}\right), \\
\left.\frac{d A}{d r}\right|_{r=r_{*}} & =0, & r_{*} & =L+O\left(u_{r, i}\right), \quad A\left(r_{*}\right)=1+O\left(u_{r, i}\right),
\end{aligned}
$$

where $\mathrm{k}$ is given by $(6.6)$. In $(2,0)$ theory we then find to the leading order a $=-\frac{N^{3}}{72}[7,19]$ (cf. (5.2)). Starting with the general $R^{2}+R^{3}$ corrected action (6.1), (6.2) we get ${ }^{28}$

$$
\begin{aligned}
\mathrm{a} & =-\mathrm{k}\left(1-7 f_{2}+14 f_{3}\right), \quad r_{*}=L\left(1-\frac{3}{5} f_{2}+\frac{2}{5} f_{3}\right), \\
f_{2} & =u_{2,1}+3\left(u_{2,2}+7 u_{2,3}\right), \\
f_{3} & =u_{3,1}-u_{3,2}-3\left(u_{3,3}-7 u_{3,4}\right)+9\left(u_{3,5}+u_{3,6}+7 u_{3,7}+49 u_{3,8}\right) .
\end{aligned}
$$

\footnotetext{
${ }^{27}$ The boundary theory defined on $S^{6}$ and thus its conformal anomaly being determined just by the coefficient of the Euler density in (1.4). The IR divergent part of the $7 \mathrm{~d}$ action evaluated on modified $\mathrm{AdS}_{7}$ solution (with log IR divergence coming from the volume factor) should then determine the UV log part of the corresponding boundary effective action. AdS solution will always be a solution of the modified gravitational equations on symmetry grounds.

${ }^{28}$ Note that if $u_{2, i}$ and $u_{3, i}$ are treated on an equal footing then it is possible to choose them so that the radius is not modified and yet the a-coefficient receives a correction: $f_{3}=\frac{3}{2} f_{2}, \quad \mathrm{a}=-\mathrm{k}\left(1+14 f_{2}\right)$. Let us note also that in the case when $R^{2}$ terms in (6.2) correspond to the square of the Weyl tensor (here $\left.d=7\right) C_{\mu \nu \lambda \rho}^{2}=$ $R_{\mu \nu \lambda \rho}^{2}-\frac{4}{d-2} R_{\mu \nu}^{2}+\frac{2}{(d-1)(d-2)} R^{2}$ we get $f_{2}=0$ as expected: in this case the $\mathrm{AdS}_{7}$ solution is not modified and the value of the action is unchanged. The same conclusion is found also for a combination of $C^{2}+C^{3}$ terms.
} 
The coefficients $\mathrm{c}_{i}$ in (6.5) and a in (6.11) can be written in a more compact form as

$$
\begin{aligned}
\mathrm{c}_{1} & =-96 \mathrm{k}\left[1-21 \bar{u}_{2,2}-\frac{5}{3} \bar{u}_{2,1}-2\left(9 u_{3,1}+23 u_{3,2}\right)+\ldots\right], \\
\mathrm{c}_{2} & =-24 \mathrm{k}\left[1-21 \bar{u}_{2,2}-\frac{37}{3} \bar{u}_{2,1}-2\left(9 u_{3,1}+7 u_{3,2}\right)+\ldots\right], \\
\mathrm{c}_{3} & =8 \mathrm{k}\left[1-21 \bar{u}_{2,2}+9 \bar{u}_{2,1}-2\left(41 u_{3,1}+31 u_{3,2}\right)+\ldots\right], \\
\mathrm{a} & =-\mathrm{k}\left[1-21 \bar{u}_{2,2}-7 \bar{u}_{2,1}+14\left(u_{3,1}-u_{3,2}\right)+\ldots\right],
\end{aligned}
$$

where we introduced the combinations

$$
\begin{aligned}
& \bar{u}_{2,1} \equiv u_{2,1}+6\left(u_{3,3}-7 u_{3,4}\right), \\
& \bar{u}_{2,2} \equiv u_{2,2}+7 u_{2,3}-6\left(u_{3,5}+u_{3,6}+7 u_{3,7}+49 u_{3,8}\right) .
\end{aligned}
$$

Thus the 4 Weyl anomaly coefficients depend only on 4 non-trivial parameters. This simplification is due to the fact that several curvature invariants in (6.4) give equivalent contributions to the on-shell action. Indeed, $\bar{u}_{2,2}$ represents the contribution of $R^{2}+R^{3}$ terms that depend just on Ricci tensor and thus renormalize the value of the cosmological constant contribution or the overall scale of the leading-order contribution to the anomaly only. The coefficient $\bar{u}_{2,1}$ represents the terms that reduce to $R_{\mu \nu \kappa \lambda}^{2}$ on the equations of motion. This ambiguity can be fixed by setting some redundant coefficients to zero. For example, we may demand that the radius is not modified, which requires according to (6.11) that $3 f_{2}=2 f_{3}$. This may be arranged by fixing a combination of parameters that does not enter the conformal anomaly coefficients.

Let us now study possible supersymmetry constraints. Computing the combinations $q_{1}$ in (1.7) and $q_{2}$ in (1.8) for the coefficients in (6.13) we find

$$
\begin{aligned}
& q_{1}=\mathrm{c}_{1}-2 \mathrm{c}_{2}+6 \mathrm{c}_{3}=3072 \mathrm{k}\left(-u_{3,1}+\frac{1}{4} u_{3,2}\right), \\
& q_{2}=\mathrm{c}_{1}-4 \mathrm{c}_{2}=3072 \mathrm{k}\left(-\frac{1}{3} \bar{u}_{2,1}+u_{3,2}\right) .
\end{aligned}
$$

We observe that $q_{1}$ does not depend on $u_{2, i}$, i.e. all $R^{2}$ corrections obey $q_{1}=0$. They should thus preserve the $(1,0)$ supersymmetry of the boundary theory [15]. Indeed, the $R^{2}$ terms admit supersymmetric extension (such corrections appear, e.g., in compactifications that break half of maximal supersymmetry). The $R^{3}$ corrections consistent with $(1,0)$ supersymmetry should obey

$$
(1,0): \quad u_{3,2}=4 u_{3,1} .
$$

Demanding both $q_{1}$ and $q_{2}$ to vanish that should correspond to the maximal supersymmetry case, i.e. to the $(2,0) 6 \mathrm{~d}$ boundary theory, gives ${ }^{29}$

$$
(2,0): \quad u_{3,2}=4 u_{3,1}, \quad \bar{u}_{2,1}=12 u_{3,1} .
$$

\footnotetext{
${ }^{29}$ The terms $I_{2,2}, I_{2,3}, I_{3,5}, I_{3,6}, I_{3,7}, I_{3,8}$ in (6.3), (6.4) that on the leading-order equations of motion $\left(R_{\mu \nu}=\Lambda g_{\mu \nu}\right)$ renormalize only the cosmological constant term should also preserve the $(2,0)$ supersymmetry - indeed, their coefficients do not appear in $q_{2}$. The terms that break $(2,0)$ supersymmetry even in the absence of $I_{3,1}, I_{3,2}$ and are $I_{2,1}$ and also $I_{3,3}$ and $I_{3,4}$ that reduce to $I_{2,1}$ on the leading-order equations of motion.
} 


\subsection{Special $(1,0)$ case: Lovelock action}

Let us study in more detail the $(1,0)$ case $(6.18)$. This constraint implies that the two irreducible curvature invariants $I_{3,2}$ and $I_{3,1}$ appear in the same combination as in the Euler density $E_{6}$. Indeed, introducing the higher order Euler densities ${ }^{30}$

$$
E_{2 p} \equiv \delta_{\mu_{1} \mu_{2} \cdots \mu_{2 p-1} \mu_{2 p}}^{\nu_{1} \nu_{2} \cdots \nu_{2}-1 \nu_{2 p}} R_{\nu_{1} \nu_{2}}^{\mu_{1} \mu_{2}} \cdots R_{\nu_{2 p-1} \nu_{2 p}}^{\mu_{2 p-1} \mu_{2 p}}=\frac{1}{(d-2 p) !} \epsilon_{d} \epsilon_{d} R \cdots R
$$

one finds using the explicit expressions for $E_{2 p}=u_{p, i} I_{p, i}$ in the bases (6.3) and (6.4) ${ }^{31}$ that choosing $\Delta \mathcal{L}$ in (6.2) in the special " $E_{4}+E_{6}$ " form we get

$$
\begin{aligned}
\Delta \mathcal{L}_{E} & =\frac{1}{4} L^{2} \mathrm{u}_{2} E_{4}+\frac{1}{16} L^{4} \mathrm{u}_{3} E_{6}, \\
u_{2, i} & =\mathrm{u}_{2} \cdot(1,-4,1), \quad u_{3, i}=\mathrm{u}_{3} \cdot\left(1,4,12, \frac{3}{2}, 12,8,-6, \frac{1}{2}\right) .
\end{aligned}
$$

Here the coefficients in (6.13) are

$$
\bar{u}_{2,1}=\mathrm{u}_{2}+9 \mathrm{u}_{3}, \quad \bar{u}_{2,2}=3 \mathrm{u}_{2}-15 \mathrm{u}_{3}, \quad u_{3,2}=4 u_{3,1}=4 \mathrm{u}_{3} .
$$

For the particular case of the action (6.1) with $\Delta \mathcal{L}$ in the Lovelock form (6.21) treated as a complete theory (i.e. not expanding in $\mathrm{u}_{2}$ and $\mathrm{u}_{3}$ and not including higher curvature terms) the corresponding conformal anomaly coefficients were found in [60-62]. They can be expressed as [62]

$$
\begin{array}{ll}
a=-\mathrm{kf}^{-5 / 2}\left(1-40 \mathrm{u}_{2} \mathrm{f}+180 \mathrm{u}_{3} \mathrm{f}^{2}\right), & \mathrm{c}_{1}=-96 \mathrm{kf}^{-5 / 2}\left(1-\frac{104}{3} \mathrm{u}_{2} \mathrm{f}+68 \mathrm{u}_{3} \mathrm{f}^{2}\right), \\
\mathrm{c}_{2}=-24 \mathrm{kf}^{-5 / 2}\left(1-\frac{136}{3} \mathrm{u}_{2} \mathrm{f}+100 \mathrm{u}_{3} \mathrm{f}^{2}\right), & \mathrm{c}_{3}=8 \mathrm{kf}^{-5 / 2}\left(1-24 \mathrm{u}_{2} \mathrm{f}+36 \mathrm{u}_{3} \mathrm{f}^{2}\right) .
\end{array}
$$

Here $\mathrm{f}$ is a function of $\mathrm{u}_{2}, \mathrm{u}_{3}$ given by a root of a cubic equation below. It enters the expression for the renormalized AdS radius $r_{*}{ }^{32}$

$$
r_{*}=\mathrm{f}^{-1 / 2} L, \quad \mathrm{f}-12 \mathrm{u}_{2} \mathrm{f}^{2}+12 \mathrm{u}_{3} \mathrm{f}^{3}=1,
$$

which generalizes the linearized expression in (6.11). The expressions (6.2) agree with the leading order results in (6.13) after one uses (6.21), (6.23) and expands to linear order in $\mathrm{u}_{2}, \mathrm{u}_{3}$.

From (6.2) we find that the combinations of coefficients in (6.16), (6.17) are

$$
q_{1}=0, \quad q_{2}=-1024 \mathrm{kf}^{-5 / 2}\left(\mathrm{u}_{2} \mathrm{f}-3 \mathrm{u}_{3} \mathrm{f}^{2}\right),
$$

\footnotetext{
${ }^{30}$ Here $\delta_{\mu_{1} \ldots \mu_{n}}^{\nu_{1} \ldots \nu_{n}}=n ! \delta_{\left[\mu_{1}\right.}^{\nu_{1}} \ldots \delta_{\left.\mu_{n}\right]}^{\nu_{n}}$ and we assume Euclidean signature, i.e. $\epsilon_{d} \epsilon_{d}=(d-2 p) ! \delta_{\ldots} \ldots$. Note that $E_{6}$ used in [7] was of the opposite sign.

${ }^{31}$ For summary of properties of $R^{3}$ invariants see, e.g., $[64,65]$. Note, in particular, that if we introduce $J_{1}=I_{3,1}, J_{2}=R_{\mu \nu \kappa \lambda} R^{\rho \nu \sigma \lambda} R_{\rho \mu \sigma \kappa}, J_{3}=-I_{3,2}$ then $J_{2}=J_{3}+\frac{1}{4} J_{1}$. Also, in the notation used in [7] we have $A_{16}=J_{1}, A_{17}=J_{2}, A_{15}=-I_{3,3}$, etc.

${ }^{32}$ The action evaluated on $\mathrm{AdS}_{7}$ is proportional to $r^{7}\left[-42 / r^{2}+30+70 \times 12\left(\mathrm{u}_{2} r^{2}-3 \mathrm{u}_{3}\right) / r^{6}\right]$ which is extremized on the solution of the above equation for $\mathrm{f} \equiv 1 / r^{2}$ (we set here $L=1$ ).
} 
i.e. $(1,0)$ supersymmetry is preserved but $(2,0)$ is broken in general. Compared to the general case in (6.11), (6.13) discussed above where the a, $\mathrm{c}_{i}$ coefficients depended on 4 parameters and one more parameter controlled the deformation of the AdS radius, the Lovelock Lagrangian (6.21) depending on just 2 parameters is too constrained. We may introduce an extra term (for example, scalar $R^{2}$ or $R^{3}$ ) to arrange that the AdS radius is not renormalized, i.e. $\mathrm{f}=1$, and then the coefficients in (6.2) will be linear in $\mathrm{u}_{2}, \mathrm{u}_{3}$.

\section{$6.3 \quad(2,0)$ case}

Let us now go back to the condition (6.19) that should correspond to the $(2,0)$ theory. Assuming (6.19) we get from (6.13) (dots stand for possible higher order terms)

$$
\begin{aligned}
\mathrm{a} & =-\mathrm{k}\left(1-21 \bar{u}_{2,2}-126 u_{3,1}\right)+\ldots, & \mathrm{c} & =-\mathrm{k}\left(1-21 \bar{u}_{2,2}-222 u_{3,1}\right)+\ldots, \\
\mathrm{c}_{1} & =96 \mathrm{c}, & \mathrm{c}_{2} & =24 \mathrm{c}, \quad \mathrm{c}_{3}=-8 \mathrm{c} .
\end{aligned}
$$

Assuming as in [52] that we may use the purely-gravitational $7 \mathrm{~d}$ action (6.1) for the description of the $S^{4}$ reduction of $11 \mathrm{~d}$ effective action (i.e. assuming that possible flux-dependent terms may be mimicked by "redundant" curvature invariants) we may apply these expressions to the $(2,0)$ theory where we expect to find that (see $(5.2),(5.3))$

$$
\mathrm{a}=-\frac{1}{288}\left[4 N^{3}\left(1-\frac{9}{16 N^{2}}\right)+\frac{7}{4}\right], \quad \mathrm{c}=-\frac{1}{288}\left[4 N^{3}\left(1-\frac{3}{4 N^{2}}\right)+1\right] .
$$

As already mentioned in section 5 , here the $N^{3}$ terms come for leading supergravity part in (6.1) [19], the constant terms (equivalent to the contribution of one tensor multiplet) come from 1-loop 11d supergravity contribution [43] while the $1 / N^{2}$ terms in brackets should represent the exact contribution of $R^{4}$ curvature corrections to the 11d M-theory action. These terms follow from (6.27) with $\mathrm{k}$ given by (6.7) if

$$
\bar{u}_{2,2}=\frac{27}{1792 N^{2}}, \quad u_{3,1}=\frac{1}{512 N^{2}} .
$$

In addition, we may also arrange that the AdS radius is not renormalized by fixing one of the "redundant" $u_{r, i}$ coefficients in (6.12) so that $3 f_{2}=2 f_{3}$. That should be important to ensure that a and $\mathrm{c}$ do not receive further $1 / N$ corrections (depending on higher powers of $\left.u_{r, i} \sim \frac{1}{N^{2}}\right)$, cf. (6.25).

In the case of special Lovelock action (6.21) the $(2,0)$ condition $q_{2}=0$ in (6.26) implies that the coefficients $\mathrm{u}_{2}$ and $\mathrm{u}_{3}$ should be related as $\mathrm{u}_{2}=3 \mathrm{fu}_{3}$ and then $\mathrm{f}$ solving cubic equation in (6.25) becomes a non-polynomial function of $\mathrm{u}_{3}$. The same will then apply to the conformal anomaly coefficients in (6.2). To avoid this complication we may assume that adding an extra scalar $R^{2}$ or $R^{3}$ invariant to (6.21) one can independently arrange to have $\mathrm{f}=1$. In that case we will have $\mathrm{u}_{2}=3 \mathrm{u}_{3}$, and then (6.21) will take the form

$$
\Delta \mathcal{L}_{E}=\frac{1}{4} L^{2} \mathrm{u}_{2}\left(E_{4}+\frac{1}{12} L^{2} E_{6}\right), \quad \mathrm{u}_{2}=3 u_{3,1}=\frac{3}{512 N^{2}},
$$

where we fixed the value of $\mathrm{u}_{2}$ to match the value of $\mathrm{c}$ in (6.29) (see (6.23), (6.30)). Note that this correction by itself will not reproduce the expected value of a in (6.29) for any $\mathrm{u}_{2}$ but the a-coefficient will get a contribution from an extra $R^{2}$ or $R^{3}$ invariant (cf. (6.13)). 
The crucial point is that irreducible $R^{3}$ invariants in (6.4) should form the $E_{6}$ combination in order to be consistent with supersymmetry and thus potentially describe subleading correction to the conformal anomaly of $(2,0)$ theory was originally pointed out in [52]. There it was suggested that a particular $R^{4}$ super-invariant term in $11 \mathrm{~d}$ action translates upon compactification on $S^{4}$ into a combination of $E_{6}$ and $E_{4}$ corrections to 7 d action which should thus produce subleading contributions to the Weyl anomaly coefficients of $(2,0)$ theory. While the discussion in [52] was admittedly heuristic (other possible 4-form and Ricci tensor terms were ignored and the contribution to the conformal anomaly coming from the $E_{4}$ term in $7 \mathrm{~d}$ action was not included) the key role of the $E_{6}$ invariant was noticed.

To recall, the starting point in [52] was the $11 \mathrm{~d} R^{4}$ invariant that involves the $8 \mathrm{~d}$ Euler density $E_{8}$ factor (see (6.20)). If $M^{11}=M^{7} \times S^{4}$ then splitting indices in $4+7$ way and performing combinatorical count one finds that ${ }^{33}$

$$
\begin{aligned}
E_{8}\left(M^{7} \times S^{4}\right) & =4 E_{6}\left(M^{7}\right) E_{2}\left(S^{4}\right)+6 E_{4}\left(M^{7}\right) E_{4}\left(S^{4}\right) \\
& =\frac{3 \times 2^{5}}{l^{2}} E_{6}\left(M^{7}\right)+\frac{3^{2} \times 2^{7}}{l^{4}} E_{4}\left(M^{7}\right)
\end{aligned}
$$

where $l$ is the radius of $S^{4}$ which in the case of $\operatorname{AdS}_{7} \times S^{4}$ solution ${ }^{34}$ is $l=\frac{1}{2} L$ where $L$ is the scale of $\mathrm{AdS}_{7}$. Then the resulting combination in (6.32) becomes

$$
E_{8}\left(M^{7} \times S^{4}\right)=\frac{3^{2} \times 2^{10}}{L^{4}}\left(E_{4}+\frac{1}{24} L^{2} E_{6}\right)
$$

Note that this combination of $E_{4}$ and $E_{6}$ is different from the one in (6.31) that was apparently required to reproduce the right $(2,0)$ ratio of the $c_{i}$-coefficients. This is not, however, a contradiction as some extra "reducible" (Ricci-tensor dependent) $R^{2}$ or $R^{3}$ terms are required to be added to both (6.31) or (6.33) in order to ensure that the leading-order expression for the $\mathrm{AdS}_{7}$ radius is not corrected (which was an assumption in [52]). As we have explained above, starting with a general enough combination of $R^{2}+R^{3}$ terms one can reproduce the values of the conformal anomaly coefficients in (6.29) and also ensure that the AdS radius is not renormalized, implying that the resulting coefficients do not get further $1 / N^{2}$ corrections.

One may attempt to repeat similar considerations for less supersymmetric models corresponding, e.g., to different choices of $M^{4}$ compactification space or adding extra fluxes (see [66] and refs. there). In addition to changing the coefficient of the $N^{3}$ in the leading terms as the AdS volume will be different that will also lead to subleading $R^{2}$ corrections that may have coefficients $N^{2}$ instead of $N$ as in [58].

\footnotetext{
${ }^{33}$ Here we correct the coefficient of the second term which was having an extra factor of 2 in [52]. In this second term we have to assign a 7 d-type index to two out of four Riemann tensors. This may be done in $\left(\begin{array}{l}4 \\ 2\end{array}\right)=6$ ways, or $3 \times 4$ divided by 2 ! because of the symmetry of the two $R$ factors.

${ }^{34}$ M5 brane metric is $d s^{2}=h^{1 / 3}(y)\left(d x_{m} d x_{m}\right)+h^{-2 / 3}(y) d y_{k} d y_{k}$ where $h=1+\frac{q}{y^{3}}, \quad d y_{k} d y_{k}=d y^{2}+$ $y^{2} d S_{4}$ so that in the near-horizon limit we get $d s^{2}=q\left(y^{-1} d x_{m} d x_{m} / y+y^{-2} d y^{2}+d S_{4}\right)$. The AdS part is $4 z^{-2}\left(d x_{m} d x_{m}+d z^{2}\right)$ were $y=\frac{1}{4} z^{2}$ so that the $\mathrm{AdS}_{7}$ radius is twice the radius of $S_{4}$.
} 


\section{Acknowledgments}

We thank M. Günaydin, J. Heckman, C. Herzog, K. Intriligator, A. Parnachev, A. Patrushev and R. Roiban for useful discussions, comments and questions. The work of A.A.T. is supported by the ERC Advanced grant No.290456, the STFC grant ST/J0003533/1 and also by the Russian Science Foundation grant 14-42-00047 associated with Lebedev Institute.

\section{A Conformal anomaly $V^{(1,0)}$ multiplet on Ricci flat background}

To provide information about $c_{i}$ coefficients for higher derivative superconformal vector multiplet $V^{(1,0)}$, here we compute the conformal anomaly (1.4) for its fields in (2.2) on Ricci flat background using the results of [7]. For a scalar, we have (using (1.5) and dropping total derivatives)

$$
7 ! A_{6}(\varphi)=\left.\left(\frac{5}{72} E_{6}-\frac{28}{3} I_{1}+\frac{5}{3} I_{2}+2 I_{3}\right)\right|_{R_{m n}=0} \rightarrow \frac{28}{9} I_{1}+\frac{17}{9} I_{2}
$$

For the standard 6d Majorana-Weyl fermion

$$
7 ! A_{6}(\psi)=\left.\left(\frac{191}{288} E_{6}-\frac{224}{3} I_{1} 8 I_{2}+10 I_{3}\right)\right|_{R_{m n}=0} \rightarrow \frac{70}{9} I_{1}+\frac{29}{9} I_{2}
$$

From the explicit form of the 4-derivative vector field $V^{(4)}$ kinetic operator on a curved background given in [8] one can show that the corresponding partition function on a Ricci flat background has the form

$$
Z\left(V^{(4)}\right)=\left[\frac{\left(\operatorname{det} \Delta_{0}\right)^{3}}{\left(\operatorname{det} \Delta_{1}\right)^{2}}\right]^{1 / 2}
$$

where $\Delta_{0,1}=-\nabla^{2}$ are Laplacians defined on scalars and vectors respectively. The contribution of $\Delta_{1}$ can be found in [7]:

$$
\left.7 ! A_{6}\left(\Delta_{1}\right)\right|_{R_{m n}=0}=-\frac{112}{3} I_{1}-\frac{50}{3} I_{2} .
$$

Taking into account that the fermion in $V^{(1,0)}$ multiplet in $(2.2)$ has $\not^{3}$ kinetic term we find for the total contribution

$$
\begin{aligned}
\left.A_{6}\left(V^{(1,0)}\right)\right|_{R_{m n}=0} & =\left.\left(3 A_{6}(\varphi)+2 \cdot 3 \cdot A_{6}(\psi)+\left[2 A_{6}\left(\Delta_{1}\right)-3 A_{6}(\varphi)\right]\right)\right|_{R_{m n}=0} \\
& =-\frac{1}{360}\left(2 I_{1}+I_{2}\right)=-\frac{1}{11520} E_{6} .
\end{aligned}
$$

Comparing to the general expression (1.6) this implies that the expected $(1,0)$ supersymmetry relation (1.7) is satisfied. Furthermore, using the known [8] value of a-coefficient in table 2 in we conclude that $\mathrm{c}_{1}+4 \mathrm{c}_{2}=\frac{62}{45}$, in agreement with the values of $\mathrm{c}_{1}, \mathrm{c}_{2}$ in $(2.3)$. 


\section{B Chiral anomalies of $\mathrm{CSG}_{p}$ supermultiplet}

Here we shall discuss the computation of the chiral R-symmetry anomaly and the mixed R-symmetry - gravitational anomaly coefficients $\alpha$ and $\beta$ in (1.11) for the $\mathrm{CSG}_{p}$ multiplet considered in section 4 , demonstrating the relation (4.6).

\section{B.1 General $p>2$ case}

Let us start with coefficient $\alpha$ of R-symmetry anomaly. We shall consider the anomaly of $\mathrm{SU}(2)$ subgroup of USp(4) R-symmetry of fields in table 4. To fix normalizations, for a positive-chirality MW fermion $\psi^{+}$or $\frac{1}{2}^{+}$in a representation $\mathbf{d}$ of $\mathrm{SU}(2)_{R}$ we have (see, e.g., [67])

$$
\mathcal{I}_{8}\left(\frac{1}{2}^{+}\right)=-\frac{1}{48} \operatorname{tr} F^{4}=-\frac{1}{3} Q_{\mathbf{d}}^{(4)}\left[c_{2}\left(\mathrm{SU}(2)_{R}\right)\right]^{2}
$$

where $Q_{\mathbf{d}}^{(4)}$ is the following sum of 4 -th powers of $\mathrm{U}(1)$ charges over the representation $\mathbf{d}$ (cf. $(3.3))$

$$
Q_{\mathbf{d}}^{(4)}=\sum_{k=0}^{d-1}\left(-\frac{d-1}{2}+k\right)^{4}=\frac{1}{240} d\left(d^{2}-1\right)\left(3 d^{2}-7\right) .
$$

Thus the corresponding $\alpha$ coefficient in (1.11) is

$$
\alpha\left(\frac{1}{2}^{+}\right)=-8 Q_{\mathbf{d}}^{(4)}
$$

The standard (gauge-invariant, non-conformal) MW gravitino contributes to the gauge anomaly as 5 times the contribution of a MW fermion [24]. To find the anomaly of a conformal massive (non-gauge) gravitino $\psi_{m}$ we need to add, as in (4.3), the contribution of two MW fermions, i.e.

$$
\mathcal{I}_{8}\left(\psi_{m}^{+}\right)=\mathcal{I}_{8}\left(\frac{3}{2}^{+}\right)+2 \mathcal{I}_{8}\left(\frac{1}{2}^{+}\right)=7 \mathcal{I}_{8}\left(\frac{1}{2}^{+}\right), \quad \alpha\left(\psi_{m}^{+}\right)=-56 Q_{\mathbf{d}}^{(4)}
$$

For a selfdual non-gauge 3 -form in the same representation of $\mathrm{SU}(2)_{R}$ the required value appears to $\mathrm{be}^{35}$

$$
\alpha\left(A_{m n k}^{+}\right)=-8 \alpha\left(\frac{1}{2}^{+}\right)=64 Q_{\mathbf{d}}^{(4)}
$$

Given a representation $[a, b]$ of $\mathrm{USp}(4)$ we can decompose it into representations $\left(\mathbf{d}_{\mathbf{1}}, \mathbf{d}_{\mathbf{2}}\right)$ of $\mathrm{SU}(2)_{L} \times \mathrm{SU}(2)_{R}$ and then identify the right factor with the $\mathrm{SU}(2)$ R-symmetry subgroup the anomalies of which we are to compute. The decomposition is [68]

$$
[a, b]=\sum N_{d_{1}, d_{2}}^{a, b}\left(\mathbf{d}_{\mathbf{1}}, \mathbf{d}_{2}\right)
$$

\footnotetext{
${ }^{35}$ One should be able express the anomaly of the antisymmetric tensor in terms of a spinor field anomaly as in $4 \mathrm{~d}$ case [28].
} 
where the multiplicites $N_{d_{1}, d_{2}}^{a, b}$ are determined by ${ }^{36}$

$$
\sum N_{d_{1}, d_{2}}^{a, b} n^{d_{1}-1} m^{d_{2}-1}=[(1-a n)(1-a m)(1-b)(1-b n m)]^{-1} .
$$

For a USp(4) representation $[a, b]$ we get

$$
\begin{aligned}
Q_{[a, b]}^{(4)}= & \sum N_{d_{1}, d_{2}}^{a, b} d_{1} Q_{\mathbf{d}_{2}}^{(4)} \\
= & \frac{1}{6720}(a+1)(b+1)(a+b+2)(a+2 b+3)\left(2 a^{4}+8 a^{3} b+16 a^{3}+14 a^{2} b^{2}\right. \\
& \left.+52 a^{2} b+29 a^{2}+12 a b^{3}+64 a b^{2}+74 a b-12 a+6 b^{4}+36 b^{3}+44 b^{2}-30 b\right) .
\end{aligned}
$$

Using the above relations, the total contribution to $\alpha$ coefficient from all the fields of $\mathrm{CSG}_{p}$ multiplet in table 4 is found to be

$$
\begin{aligned}
\alpha\left(\mathrm{CSG}_{p}\right)= & -8\left(Q_{[1, p-1]}^{(4)}-Q_{[3, p-3]}^{(4)}+Q_{[3, p-4]}^{(4)}-Q_{[1, p-4]}^{(4)}\right) \\
& -56\left(Q_{[1, p-2]}^{(4)}-Q_{[1, p-3]}^{(4)}\right)+64\left(Q_{[0, p-1]}^{(4)}-Q_{[0, p-3]}^{(4)}\right) \\
= & -2[6 p(p-1)+1],
\end{aligned}
$$

which is the value quoted in (4.7).

Similar analysis can be repeated for the coefficient $\beta$ of the mixed R-symmetry gravitational anomaly in (1.11). The analog of (B.1) here is

$$
\mathcal{I}_{8}\left(\frac{1}{2}^{+}\right)=-\frac{1}{192} \operatorname{tr} F^{2} \operatorname{tr} R^{2}=\frac{1}{24} Q_{\mathrm{d}}^{(2)} c_{2}\left(\mathrm{SU}(2)_{R}\right) \mathrm{p}_{1}
$$

where $Q_{\mathbf{d}}^{(2)}$ is the sum of squares of $\mathrm{U}(1)$ charges over an $\mathrm{SU}(2)_{R}$ representation of dimension $d$

$$
Q_{\mathbf{d}}^{(2)}=\sum_{k=0}^{d-1}\left(-\frac{d-1}{2}+k\right)^{2}=\frac{1}{12} d\left(d^{2}-1\right) .
$$

Thus for a MW spinor in the representation $\mathbf{d}$ (cf. (1.11))

$$
\beta\left(\frac{1}{2}^{+}\right)=-Q_{\mathbf{d}}^{(2)}
$$

The standard MW gravitino contributes to the mixed anomaly with a factor - 19 compared to the MW spinor [24]. Again, a massive conformal gravitino requires the addition of two extra MW spinor contributions, so that the total $\beta$ coefficient for a positive chirality MW conformal massive gravitino in representation $\mathbf{d}$ of $\mathrm{SU}(2)_{R}$ is

$$
\beta\left(\psi_{m}^{+}\right)=17 Q_{\mathbf{d}}^{(2)}
$$

\footnotetext{
${ }^{36}$ For example, the USp(4) representations of states in the conformal supergravity multiplet ( $p=2$ level in table 4) decompose as follows

$$
[2,0] \rightarrow(\mathbf{1}, \mathbf{3})+(\mathbf{2}, \mathbf{2})+(\mathbf{3}, \mathbf{1}), \quad[1,0] \rightarrow(\mathbf{1}, \mathbf{2})+(\mathbf{2}, \mathbf{1}), \quad[0,0] \rightarrow(\mathbf{1}, \mathbf{1})
$$$$
[0,2] \rightarrow(\mathbf{1}, \mathbf{1})+(\mathbf{2}, \mathbf{2})+(\mathbf{3}, \mathbf{3}), \quad[1,1] \rightarrow(\mathbf{1}, \mathbf{2})+(\mathbf{2}, \mathbf{1})+(\mathbf{2}, \mathbf{3})+(\mathbf{3}, \mathbf{2}), \quad[0,1] \rightarrow(\mathbf{1}, \mathbf{1})+(\mathbf{2}, \mathbf{2}),
$$ 


\begin{tabular}{|l|l|}
\hline$\left(\Delta ; h_{1}, h_{2}, h_{3}\right)$ & $\mathrm{USp}(4)$ \\
\hline$(4 ; 0,0,0)$ & {$[0,2]=\mathbf{1 4}$} \\
$\left(\frac{9}{2} ; \frac{1}{2}, \frac{1}{2}, \frac{1}{2}\right)$ & {$[1,1]=\mathbf{1 6}$} \\
$(5 ; 1,1,1)$ & {$[0,1]=\mathbf{5}$} \\
$(5 ; 1,0,0)$ & {$[2,0]=\mathbf{1 0}$} \\
$\left(\frac{11}{2} ; \frac{3}{2}, \frac{1}{2}, \frac{1}{2}\right)-\left(\frac{9}{2} ; \frac{1}{2}, \frac{1}{2}, \frac{1}{2}\right)$ & {$[1,0]=\mathbf{4}$} \\
$(6 ; 2,0,0)$ & {$[0,0]=\mathbf{1}$} \\
\hline
\end{tabular}

Table 6. $\mathrm{SO}(2,6) \times \mathrm{USp}(4)$ representations of fields of $(2,0)$ conformal supergravity.

The self-dual non-gauge antisymmetric tensor contribution is

$$
\beta\left(A_{m n k}^{+}\right)=16 \beta\left(\frac{1}{2}^{+}\right)=-16 Q_{\mathbf{d}}^{(2)} .
$$

Using that

$$
Q_{[a, b]}^{(2)}=\frac{1}{240}(a+1)(b+1)(a+b+2)(a+2 b+3)\left(a^{2}+2 a b+4 a+2 b^{2}+6 b\right),
$$

the total contribution to $\beta$ from the $\mathrm{CSG}_{p}$ multiplet in table 4 is found to be

$$
\begin{aligned}
\beta\left(\mathrm{CSG}_{p}\right)= & -\left(Q_{[1, p-1]}^{(2)}-Q_{[3, p-3]}^{(2)}+Q_{[3, p-4]}^{(2)}-Q_{[1, p-4]}^{(2)}\right) \\
& +17\left(Q_{[1, p-2]}^{(2)}-Q_{[1, p-3]}^{(2)}\right)-16\left(Q_{[0, p-1]}^{(2)}-Q_{[0, p-3]}^{(2)}\right) \\
= & -[6 p(p-1)+1]
\end{aligned}
$$

in agreement with (4.7).

As a check on normalizations used above we can re-derive the anomaly polynomial of $\mathrm{V}_{p}^{(1,0)}$ multiplet discussed in section 3. According to table 3, the fields that contribute to chiral anomalies there are a MW spinor $\frac{1}{2}^{+}$in the $\mathrm{SU}(2)_{R}$ representation $\mathbf{p}$ and a MW spinor $\frac{1}{2}^{-}$in the representation $\mathbf{p}-\mathbf{2}$, so that we find

$$
\mathrm{V}_{p}^{(1,0)}: \quad \alpha=-8\left(Q_{\mathbf{p}}^{(4)}-Q_{\mathbf{p}-2}^{(4)}\right)=-(p-1)^{4}, \quad \beta=-\left(Q_{\mathbf{p}}^{(2)}-Q_{\mathbf{p}-2}^{(2)}\right)=-\frac{1}{2}(p-1)^{2},
$$

in agreement with (3.4).

\section{B.2 (2,0) conformal supergravity}

The above discussion applied for $p>2$. In the $p=2$ case of table 4 corresponding to the $(2,0)$ conformal supergravity multiplet the gravitino is massless and thus requires a special treatment. We recall the conformal representations of the corresponding fields in table 6 . Here in the gravitino entry we explicitly indicated the subtraction of the contribution of the gauge degree of freedom which is the fermion of the same chirality. 


\begin{tabular}{|c|c|c|c|c|}
\hline & $G_{R}$ & $\psi^{+}$ & $A_{m n k}^{+}$ & $\psi_{m}^{+}$ \\
\hline$(2,0)$ & $\mathrm{USp}(4)$ & {$[1,1]=\mathbf{1 6}$} & {$[0,1]=\mathbf{5}$} & {$[1,0]=\mathbf{4}$} \\
\hline$(1,0)$ & $\mathrm{SU}(2)$ & $\mathbf{2}$ & $\mathbf{1}$ & $\mathbf{2}$ \\
\hline
\end{tabular}

Table 7. Chiral fields in the multiplets of $(2,0)$ and $(1,0)$ conformal supergravities. $G_{R}$ is the R-symmetry group. $[a, b]$ is the representation of USp(4) with Dynkin labels $a, b$.

The gravitational anomaly count then gives ${ }^{37}$

$$
16 \times \mathcal{I}_{8}\left(\frac{1}{2}^{+}\right)+5 \times 2 \mathcal{I}_{8}\left(T^{+}\right)+4 \times\left[\mathcal{I}_{8}\left(\frac{3}{2}^{+}\right)+\mathcal{I}_{8}\left(\frac{1}{2}^{+}\right)\right]=\frac{1}{4 !}\left(-\frac{13}{4} \mathrm{p}_{1}^{2}+13 \mathrm{p}_{2}\right) .
$$

This agrees with the general $p$ result (4.4) formally continued to $p=2$.

Similarly, we may compute the chiral anomaly $\alpha$ and $\beta$ coefficients (the middle term coefficients here are the conformal gauge-invariant gravitino ones)

$$
\begin{aligned}
& \alpha=-8 Q_{[1,1]}^{(4)}-48 Q_{[1,0]}^{(4)}+64 Q_{[0,1]}^{(4)}=-26, \\
& \beta=-Q_{[1,1]}^{(2)}+18 Q_{[1,0]}^{(2)}-16 Q_{[0,1]}^{(2)}=-13 .
\end{aligned}
$$

These are again in agreement with the formal $p=2$ continuation of (B.9) and (B.16).

\section{B.3 (1,0) conformal supergravity}

Let us now consider the chiral anomaly coefficients corresponding to $(1,0)$ conformal supergravity. For comparison, the chiral fields in the multiplets of $(2,0)$ and $(1,0)$ conformal supergravities are listed in table 7 below. It is then straightforward to find the analogs of the $(2,0)$ CSG expressions (B.17) and (B.18) in the (1,0) CSG case:

$$
\begin{aligned}
& 2 \times \mathcal{I}_{8}\left(\frac{1}{2}^{+}\right)+2 \mathcal{I}_{8}\left(T^{+}\right)+2 \times\left[\mathcal{I}_{8}\left(\frac{3}{2}^{+}\right)+\mathcal{I}_{8}\left(\frac{1}{2}^{+}\right)\right]=\frac{1}{4 !}\left(-\frac{107}{80} \mathrm{p}_{1}^{2}+\frac{101}{20} \mathrm{p}_{2}\right), \\
& \alpha=-8 Q_{\mathbf{2}}^{(4)}-48 Q_{\mathbf{2}}^{(4)}+64 Q_{\mathbf{1}}^{(4)}=-7, \quad \beta=-Q_{\mathbf{2}}^{(2)}+18 Q_{\mathbf{2}}^{(2)}-16 Q_{1}^{(2)}=\frac{17}{2} .
\end{aligned}
$$

Thus the set of 4 chiral anomaly coefficients here is

$$
\vec{\alpha}\left(\mathrm{CSG}^{(1,0)}\right)=\left(-7, \frac{17}{2},-\frac{107}{80}, \frac{101}{20}\right) .
$$

Applying the relation (1.13) of [1] to compute the a-coefficient we get

$$
\mathrm{a}\left(\mathrm{CSG}^{(1,0)}\right)=\frac{797}{3840}
$$

\footnotetext{
${ }^{37}$ Here in the conformal gravitino contribution we subtract the $\gamma$-trace degree of freedom (which is the opposite chirality fermion) from the contribution of the standard non-conformal gravitino $\frac{3}{2}^{+}$, cf. (4.3). The antisymmetric tensor is non-gauge one so its contribution is twice that of the gauge-invariant one in (4.1).
} 
which agrees with the value found in [8] by an independent method. This provides a non-trivial check of consistency of the chiral anomaly values (B.20) and also of the relation (1.13).

Using the relations (1.15) we may now compute the corresponding $\mathrm{c}_{i}$-coefficients:

$$
\mathrm{CSG}^{(1,0)}: \quad \mathrm{c}_{1}=\frac{157}{9}, \quad \mathrm{c}_{2}=\frac{781}{180}, \quad \mathrm{c}_{3}=-\frac{263}{180} .
$$

One may ask if there are any combinations of $(1,0)$ multiplets $\mathrm{CSG}^{(1,0)}+k_{1} S^{(1,0)}+k_{2} T^{(1,0)}+$ $k_{3} V^{(1,0)}$ that are free of all chiral (and thus also of conformal) anomalies. The answer turns out to be negative. The chiral gravitational anomalies cancel $(\gamma=\delta=0)$ for $k_{2}=10, \quad k_{3}=k_{1}-13$.

\section{Casimir energy of 6d supermultiplets}

One may wonder if the expression for the Casimir energy $E_{c}$ on $S^{5}$ for $(1,0)$ superconformal $6 \mathrm{~d}$ theories can also be expressed, like the conformal anomaly coefficients $\left(\mathrm{a}, \mathrm{c}_{i}\right)$ in (1.14), (1.15) in terms of the chiral anomaly coefficients $\vec{\alpha}=(\alpha, \beta, \gamma, \delta)$ in (1.11). ${ }^{38}$

Indeed, $E_{c}$ is determined by the $T_{00}$ component of stress tensor and should thus be related [71-73] to the a-coefficient and also to a combination of total derivative term coefficients in conformal anomaly. The latter may be expected to be more constrained in the supersymmetric case and may again be related to the chiral anomaly coefficients. One should note, however, that, in general, $E_{c}$ (and the derivative terms in the trace anomaly) are scheme-dependent so that a relation to scheme-independent chiral anomaly coefficients may hold only in a particular "supersymmetric" scheme.

The Casimir energies for individual 6d conformal fields can be computed as in [43]. Assuming that $E_{c}$ (being related to the trace of stress tensor) may be given by a linear combination of the chiral anomaly coefficients and using particular examples of $(1,0)$ supermultiplets as data points we have found the following expression for $E_{c}$ in terms of the coefficients in in the anomaly polynomial (1.11)

$$
E_{c}=-\frac{1}{4}\left(\alpha-\frac{31}{24} \beta+\frac{5}{3} \gamma+\frac{29}{48} \delta\right) \text {. }
$$

Note that in contrast to the conformal anomaly coefficients in (1.13), (1.15) $E_{c}$ depends not only on $\alpha-\beta$ but also on $\alpha+\beta$. This relation is in agreement with the values for $E_{c}$ for particular $(1,0)$ multiplets in $(2.1),(2.2),(3.1)$ which can be found directly

$$
\begin{aligned}
E_{c}\left(S^{(1,0)}\right) & =-\frac{37}{3840}, & E_{c}\left(T^{(1,0)}\right)=-\frac{71}{1280}, & E_{c}\left(\mathrm{CSG}^{(1,0)}\right)=\frac{16471}{3840}, \\
E_{c}\left(\mathrm{~V}_{p}^{(1,0)}\right) & =\frac{1}{4}(p-1)^{4}-\frac{31}{192}(p-1)^{2}+\frac{37}{3840}, & & \\
E_{c}\left(\mathrm{CSG}_{p}\right) & =-2[6 p(p-1)+1] E_{c}\left(T^{(2,0)}\right), & E_{c}\left(T^{(2,0)}\right) & =-\frac{25}{384}
\end{aligned}
$$

\footnotetext{
${ }^{38}$ Here we are consider the standard Casimir energy, not the "supersymmetric" one in [69, 70] and refs. there.
} 
In the $(2,0)$ case where we expect to have $\beta=4 \gamma=-\delta$ (see $(1.17),(1.18)$ ) so that acoefficient is given by (1.19) we get for $E_{c}$ in (C.1)

$$
E_{c}=-\frac{1}{4}\left(\alpha+\frac{71}{48} \delta\right) .
$$

In contrast to what happens in the maximally supersymmetric case in 4 dimensions this $E_{c}$ is not directly proportional to the corresponding expression for $\mathrm{a}=-\frac{1}{72}\left(\alpha+\frac{9}{8} \delta\right)$ in (1.19). This suggests that in $6 \mathrm{~d}$ case the derivative term contribution to the relation between $E_{c}$ and a-anomaly does not vanish even in the $(2,0)$ case (cf. [72]).

Open Access. This article is distributed under the terms of the Creative Commons Attribution License (CC-BY 4.0), which permits any use, distribution and reproduction in any medium, provided the original author(s) and source are credited.

\section{References}

[1] C. Cordova, T.T. Dumitrescu and K. Intriligator, Anomalies, renormalization group flows and the a-theorem in six-dimensional $(1,0)$ theories, arXiv:1506.03807 [INSPIRE].

[2] M.J. Duff, Twenty years of the Weyl anomaly, Class. Quant. Grav. 11 (1994) 1387 [hep-th/9308075] [INSPIRE].

[3] D. Anselmi, J. Erlich, D.Z. Freedman and A.A. Johansen, Positivity constraints on anomalies in supersymmetric gauge theories, Phys. Rev. D 57 (1998) 7570 [hep-th/9711035] [INSPIRE].

[4] O. Aharony and Y. Tachikawa, A holographic computation of the central charges of $D=4$, $N=2$ SCFTs, JHEP 01 (2008) 037 [arXiv:0711.4532] [INSPIRE].

[5] L. Bonora, P. Pasti and M. Bregola, Weyl cocycles, Class. Quant. Grav. 3 (1986) 635 [INSPIRE].

[6] S. Deser and A. Schwimmer, Geometric classification of conformal anomalies in arbitrary dimensions, Phys. Lett. B 309 (1993) 279 [hep-th/9302047] [INSPIRE].

[7] F. Bastianelli, S. Frolov and A.A. Tseytlin, Conformal anomaly of $(2,0)$ tensor multiplet in six-dimensions and AdS/CFT correspondence, JHEP 02 (2000) 013 [hep-th/0001041] [INSPIRE].

[8] M. Beccaria and A.A. Tseytlin, Conformal a-anomaly of some non-unitary $6 d$ superconformal theories, JHEP 09 (2015) 017 [arXiv:1506.08727] [INSPIRE].

[9] L. Fei, S. Giombi, I.R. Klebanov and G. Tarnopolsky, Generalized F-theorem and the $\epsilon$ expansion, arXiv:1507.01960 [INSPIRE].

[10] A.A. Tseytlin, Weyl anomaly of conformal higher spins on six-sphere, Nucl. Phys. B 877 (2013) 632 [arXiv:1310.1795] [InSPIRE].

[11] S. Deser, Closed form effective conformal anomaly actions in $D \geq 4$, Phys. Lett. B 479 (2000) 315 [hep-th/9911129] [INSPIRE].

[12] H. Osborn and A.C. Petkou, Implications of conformal invariance in field theories for general dimensions, Annals Phys. 231 (1994) 311 [hep-th/9307010] [INSPIRE]. 
[13] J. Erdmenger and H. Osborn, Conserved currents and the energy momentum tensor in conformally invariant theories for general dimensions, Nucl. Phys. B 483 (1997) 431 [hep-th/9605009] [INSPIRE].

[14] F. Bastianelli, S. Frolov and A.A. Tseytlin, Three point correlators of stress tensors in maximally supersymmetric conformal theories in $D=3$ and $D=6$, Nucl. Phys. B 578 (2000) 139 [hep-th/9911135] [INSPIRE].

[15] M. Kulaxizi and A. Parnachev, Supersymmetry constraints in holographic gravities, Phys. Rev. D 82 (2010) 066001 [arXiv:0912.4244] [inSPIRE].

[16] D.M. Hofman and J. Maldacena, Conformal collider physics: energy and charge correlations, JHEP 05 (2008) 012 [arXiv:0803.1467] [INSPIRE].

[17] B.R. Safdi, Exact and numerical results on entanglement entropy in $(5+1)$-dimensional CFT, JHEP 12 (2012) 005 [arXiv:1206.5025] [INSPIRE].

[18] P. Bueno and R.C. Myers, Universal entanglement for higher dimensional cones, arXiv: 1508.00587 [INSPIRE].

[19] M. Henningson and K. Skenderis, The holographic Weyl anomaly, JHEP 07 (1998) 023 [hep-th/9806087] [INSPIRE].

[20] P.S. Howe, G. Sierra and P.K. Townsend, Supersymmetry in six-dimensions, Nucl. Phys. B 221 (1983) 331 [inSPIRE].

[21] R. Manvelyan and A.C. Petkou, $A$ note on $R$ currents and trace anomalies in the $(2,0)$ tensor multiplet in D =6 AdS/CFT correspondence, Phys. Lett. B 483 (2000) 264 [hep-th/0003017] [INSPIRE].

[22] R. Manvelyan and W. Rühl, On the supermultiplet of anomalous currents in $D=6$, Phys. Lett. B 567 (2003) 53 [hep-th/0305138] [INSPIRE].

[23] P.H. Frampton and T.W. Kephart, Explicit evaluation of anomalies in higher dimensions, Phys. Rev. Lett. 50 (1983) 1343 [Erratum ibid. 51 (1983) 232] [INSPIRE].

[24] L. Álvarez-Gaumé and E. Witten, Gravitational anomalies, Nucl. Phys. B 234 (1984) 269 [INSPIRE].

[25] B. Zumino, Y.-S. Wu and A. Zee, Chiral anomalies, higher dimensions and differential geometry, Nucl. Phys. B 239 (1984) 477 [INSPIRE].

[26] J.J. Heckman and T. Rudelius, Evidence for C-theorems in 6D SCFTs, JHEP 09 (2015) 218 [arXiv: 1506.06753] [INSPIRE].

[27] A.A. Tseytlin, On partition function and Weyl anomaly of conformal higher spin fields, Nucl. Phys. B 877 (2013) 598 [arXiv: 1309.0785] [INSPIRE].

[28] H. Romer and P. van Nieuwenhuizen, Axial anomalies in $N=4$ conformal supergravity, Phys. Lett. B 162 (1985) 290 [INSPIRE].

[29] A.V. Smilga, Chiral anomalies in higher-derivative supersymmetric $6 D$ theories, Phys. Lett. B 647 (2007) 298 [hep-th/0606139] [INSPIRE].

[30] J.J.M. Carrasco, R. Kallosh, R. Roiban and A.A. Tseytlin, On the U(1) duality anomaly and the $S$-matrix of $N=4$ supergravity, JHEP 07 (2013) 029 [arXiv:1303.6219] [INSPIRE].

[31] J. de Boer, M. Kulaxizi and A. Parnachev, $A d S_{7} / C F T_{6}$, Gauss-Bonnet gravity and viscosity bound, JHEP 03 (2010) 087 [arXiv:0910.5347] [INSPIRE]. 
[32] E.A. Ivanov and A.V. Smilga, Conformal properties of hypermultiplet actions in six dimensions, Phys. Lett. B 637 (2006) 374 [hep-th/0510273] [INSPIRE].

[33] S. Giombi, I.R. Klebanov, S.S. Pufu, B.R. Safdi and G. Tarnopolsky, AdS description of induced higher-spin gauge theory, JHEP 10 (2013) 016 [arXiv:1306.5242] [INSPIRE].

[34] S. Giombi, I.R. Klebanov and B.R. Safdi, Higher spin $A d S_{d+1} / C F T_{d}$ at one loop, Phys. Rev. D 89 (2014) 084004 [arXiv:1401.0825] [InSPIRE].

[35] M. Beccaria and A.A. Tseytlin, Higher spins in $A d S_{5}$ at one loop: vacuum energy, boundary conformal anomalies and AdS/CFT, JHEP 11 (2014) 114 [arXiv:1410.3273] [INSPIRE].

[36] S. Minwalla, Restrictions imposed by superconformal invariance on quantum field theories, Adv. Theor. Math. Phys. 2 (1998) 781 [hep-th/9712074] [INSPIRE].

[37] V.K. Dobrev, Positive energy unitary irreducible representations of $D=6$ conformal supersymmetry, J. Phys. A 35 (2002) 7079 [hep-th/0201076] [INSPIRE].

[38] J. Bhattacharya, S. Bhattacharyya, S. Minwalla and S. Raju, Indices for superconformal field theories in 3,5 and 6 dimensions, JHEP 02 (2008) 064 [arXiv:0801.1435] [INSPIRE].

[39] S. Ferrara and E. Sokatchev, Representations of $(1,0)$ and $(2,0)$ superconformal algebras in six-dimensions: massless and short superfields, Lett. Math. Phys. 51 (2000) 55 [hep-th/0001178] [INSPIRE].

[40] A. Van Proeyen, Tools for supersymmetry, in Proceedings, Spring School on Quantum Field Theory. Supersymmetries and Superstrings, Ann. U. Craiova Phys. 9 (1999) I.1 [hep-th/9910030] [INSPIRE].

[41] E.G. Gimon and C. Popescu, The operator spectrum of the six-dimensional $(1,0)$ theory, JHEP 04 (1999) 018 [hep-th/9901048] [INSPIRE].

[42] V. Kumar, D.S. Park and W. Taylor, 6D supergravity without tensor multiplets, JHEP 04 (2011) 080 [arXiv: 1011.0726] [INSPIRE].

[43] M. Beccaria, G. Macorini and A.A. Tseytlin, Supergravity one-loop corrections on $A d S_{7}$ and $A d S_{3}$, higher spins and AdS/CFT, Nucl. Phys. B 892 (2015) 211 [arXiv:1412.0489] [INSPIRE].

[44] A. Casher, F. Englert, H. Nicolai and M. Rooman, The mass spectrum of supergravity on the round seven sphere, Nucl. Phys. B 243 (1984) 173 [inSPIRE].

[45] M. Günaydin, P. van Nieuwenhuizen and N.P. Warner, General construction of the unitary representations of anti-de Sitter superalgebras and the spectrum of the $S^{4}$ compactification of eleven-dimensional supergravity, Nucl. Phys. B 255 (1985) 63 [InSPIRE].

[46] P. van Nieuwenhuizen, The complete mass spectrum of $d=11$ supergravity compactified on $S_{4}$ and a general mass formula for arbitrary cosets $M_{4}$, Class. Quant. Grav. 2 (1985) 1 [INSPIRE].

[47] E. D'Hoker and B. Pioline, Near extremal correlators and generalized consistent truncation for $A d S_{4 \mid 7} \times S^{7 \mid 4}, J H E P 07$ (2000) 021 [hep-th/0006103] [INSPIRE].

[48] E. Bergshoeff, E. Sezgin and A. Van Proeyen, $(2,0)$ tensor multiplets and conformal supergravity in D=6, Class. Quant. Grav. 16 (1999) 3193 [hep-th/9904085] [INSPIRE].

[49] E.S. Fradkin and A.A. Tseytlin, Conformal supergravity, Phys. Rept. 119 (1985) 233 [INSPIRE]. 
[50] J.A. Harvey, R. Minasian and G.W. Moore, Non-Abelian tensor multiplet anomalies, JHEP 09 (1998) 004 [hep-th/9808060] [INSPIRE].

[51] D. Freed, J.A. Harvey, R. Minasian and G.W. Moore, Gravitational anomaly cancellation for M-theory five-branes, Adv. Theor. Math. Phys. 2 (1998) 601 [hep-th/9803205] [INSPIRE].

[52] A.A. Tseytlin, $R^{4}$ terms in 11 dimensions and conformal anomaly of $(2,0)$ theory, Nucl. Phys. B 584 (2000) 233 [hep-th/0005072] [INSPIRE].

[53] C. Beem, L. Rastelli and B.C. van Rees, $\mathcal{W}$ symmetry in six dimensions, JHEP 05 (2015) 017 [arXiv: 1404.1079] [INSPIRE].

[54] K. Ohmori, H. Shimizu, Y. Tachikawa and K. Yonekura, Anomaly polynomial of general $6 d$ SCFTs, Prog. Theor. Exp. Phys. 2014 (2014) 103B07 [arXiv: 1408.5572] [inSPIRE].

[55] P. Mansfield, D. Nolland and T. Ueno, Order $1 / N^{3}$ corrections to the conformal anomaly of the $(2,0)$ theory in six-dimensions, Phys. Lett. B 566 (2003) 157 [hep-th/0305015] [INSPIRE].

[56] O.J. Ganor and A. Hanany, Small E $E_{8}$ instantons and tensionless noncritical strings, Nucl. Phys. B 474 (1996) 122 [hep-th/9602120] [INSPIRE].

[57] K. Ohmori, H. Shimizu and Y. Tachikawa, Anomaly polynomial of E-string theories, JHEP 08 (2014) 002 [arXiv:1404.3887] [INSPIRE].

[58] J. Heckman and C. Herzog, A conformal anomaly for 6d SCFT's, unpublished draft.

[59] K. Ohmori, H. Shimizu, Y. Tachikawa and K. Yonekura, $6 d N=(1,0)$ theories on $T^{2}$ and class S theories: part I, JHEP 07 (2015) 014 [arXiv: 1503.06217] [INSPIRE].

[60] J. de Boer, M. Kulaxizi and A. Parnachev, Holographic Lovelock gravities and black holes, JHEP 06 (2010) 008 [arXiv:0912.1877] [INSPIRE].

[61] J. de Boer, M. Kulaxizi and A. Parnachev, Holographic entanglement entropy in Lovelock gravities, JHEP 07 (2011) 109 [arXiv:1101.5781] [INSPIRE].

[62] L.-Y. Hung, R.C. Myers and M. Smolkin, On holographic entanglement entropy and higher curvature gravity, JHEP 04 (2011) 025 [arXiv:1101.5813] [INSPIRE].

[63] C. Imbimbo, A. Schwimmer, S. Theisen and S. Yankielowicz, Diffeomorphisms and holographic anomalies, Class. Quant. Grav. 17 (2000) 1129 [hep-th/9910267] [INSPIRE].

[64] P. van Nieuwenhuizen and C.C. Wu, On integral relations for invariants constructed from three Riemann tensors and their applications in quantum gravity,

J. Math. Phys. 18 (1977) 182 [InSPIRE].

[65] R.R. Metsaev and A.A. Tseytlin, Curvature cubed terms in string theory effective actions, Phys. Lett. B 185 (1987) 52 [INSPIRE].

[66] A. Passias, A. Rota and A. Tomasiello, Universal consistent truncation for $6 d / 7 d$ gauge/gravity duals, JHEP 10 (2015) 187 [arXiv:1506.05462] [INSPIRE].

[67] S.D. Avramis, Anomaly-free supergravities in six dimensions, Ph.D. thesis, Natl. Tech. U., Athens Greece (2006) [hep-th/0611133] [INSPIRE].

[68] M. Couture and R.T. Sharp, Reduction of enveloping algebras of low-rank groups, J. Phys. A 13 (1990) 1925. 
[69] B. Assel, D. Cassani, L. Di Pietro, Z. Komargodski, J. Lorenzen and D. Martelli, The Casimir energy in curved space and its supersymmetric counterpart, JHEP 07 (2015) 043 [arXiv: 1503.05537] [INSPIRE].

[70] N. Bobev, M. Bullimore and H.-C. Kim, Supersymmetric Casimir energy and the anomaly polynomial, JHEP 09 (2015) 142 [arXiv:1507.08553] [INSPIRE].

[71] A. Cappelli and A. Coste, On the stress tensor of conformal field theories in higher dimensions, Nucl. Phys. B 314 (1989) 707 [InSPIRE].

[72] C.P. Herzog and K.-W. Huang, Stress tensors from trace anomalies in conformal field theories, Phys. Rev. D 87 (2013) 081901 [arXiv:1301.5002] [INSPIRE].

[73] K.-W. Huang, Weyl anomaly induced stress tensors in general manifolds, Nucl. Phys. B 879 (2014) 370 [arXiv:1308.2355] [INSPIRE]. 